\title{
Global Conformal Invariance in Quantum Field Theory
}

\author{
M. Lüscher and G. Mack \\ Institut für Theoretische Physik der Universität Bern, Bern, Switzerland
}

Received October 17, 1974

\begin{abstract}
Suppose that there is given a Wightman quantum field theory (QFT) whose Euclidean Green functions are invariant under the Euclidean conformal group $\left(\mathfrak{5} \simeq \mathrm{SO}_{e}(5,1)\right.$. We show that its Hilbert space of physical states carries then a unitary representation of the universal $(\infty$-sheeted) covering group $\left(5^{*}\right.$ of the Minkowskian conformal group $\mathrm{SO}_{e}(4,2) / \mathbb{Z}_{2}$. The Wightman functions can be analytically continued to a domain of holomorphy which has as a real boundary an $\infty$-sheeted covering $\tilde{M}$ of Minkowski-space $M^{4}$. It is known that $\mathfrak{G}^{*}$ can act on this space $\tilde{M}$ and that $\tilde{M}$ admits a globally $\mathfrak{G}^{*}$-invariant causal ordering; $\tilde{M}$ is thus the natural space on which a globally $\mathfrak{G}^{*}$ - invariant local QFT could live. We discuss some of the properties of such a theory, in particular the spectrum of the conformal Hamiltonian $H=\frac{1}{2}\left(P^{0}+K^{0}\right)$.

As a tool we use a generalized Hille-Yosida theorem for Lie semigroups. Such a theorem is stated and proven in Appendix C. It enables us to analytically continue contractive representations of a certain maximal subsemigroup $\mathfrak{\Xi}$ of $\mathfrak{G}$ to unitary representations of $\mathfrak{b}^{*}$.
\end{abstract}

\section{Introduction}

Conformal invariant quantum field theory (QFT) is of interest from the point of view of constructive quantum field theory because such theories can be analyzed to a remarkable extent by nonperturbative methods, i.e. without recourse to iterative techniques $[1,2]$. One adopts the usual postulates of local QFT (Wightman axioms [3]): Spectrum condition, positivity, and locality. In addition one demands that the Euclidean Green functions are invariant under the Euclidean conformal group ${ }^{1} \mathrm{SO}_{e}(5,1)$. [The Euclidean Green functions are obtained by analytically continuing the vacuum expectation values of fields. (Wightman functions) to imaginary times.] This is the hypothesis of "weak conformal invariance" [4]. It implies that the Wightman functions are invariant under infinitesimal conformal transformations in Minkowski space.

The hypothesis of weak conformal invariance was invented because of the familiar difficulties with global conformal transformations in Minkowski space $M^{4}$. Such a transformation can take points to infinity, and it does not help either to compactify Minkowski space, i.e. add points at infinity, because the resulting manifold $M_{c}^{4}$ contains closed timelike curves and so does not admit a global causal ordering [5].

In the present paper we consider Wightman field theories with a unique vacuum, with Wightman functions that are (tempered) distributions, and whose Euclidean Green functions are conformal invariant as explained above. We show that the Hilbert space of physical states of any such theory carries a unitary representation of the $\infty$-sheeted universal covering group $\mathfrak{r}^{*}$ of the Minkowskian

\footnotetext{
1 The subscript $e$ identifies the identity component of the group.
} 
conformal group $\mathrm{SO}_{e}(4,2) / \mathbb{Z}_{2}$. This group will be called the "quantum mechanical conformal group".

A special role is played by its generator $H \equiv J_{60}=\frac{1}{2}\left(P^{0}=K^{0}\right)$, where $K^{0}$ is one of the generators of special conformal transformations [6]. It is found to have positive semi-definite spectrum $H \geqq 0$. This operator is the most convenient choice for Hamiltonian in a conformal invariant theory. It generates a noncompact 1-parameter subgroup of $\mathfrak{6}^{*}$.

We will also show that the Wightman functions can be analytically continued into a complex domain which has as a real boundary an infinitesheeted covering $\tilde{M}$ of Minkowski space. This manifold is thus the natural space on which a globally conformal invariant QFT could live. The quantum mechanical (q.m.) conformal group can act on it and it is known to admit a global conformal invariant causal ordering [7-9]. We will show that fields living on it will have conformal invariant locality properties, if they exist at all as operator valued distributions ${ }^{2}$. They transform covariantly under $\mathbf{6}^{*}$. In contrast with Euclidean fields (where they exist) they do not transform irreducibly though, because elements $Z \in \mathbb{Z} \subset$ center of $\mathfrak{6}^{*}$ act on them in a nontrivial way in general. As Schroer and Swieca have pointed out recently [10], this implies that the unitary representation of the q.m. conformal group is not in general a ray representation of $\mathrm{SO}_{e}(4,2) / \mathbb{Z}_{2}$, nor is there a superselection rule which would rectify this. This is just another aspect of the fact that conformal symmetry is really not a symmetry of Minkowski space $M^{4}$, but of the larger "superworld" $\tilde{M}$.

The Minkowski space $M^{4}$ may be identified with a paracompact part of the superworld $\tilde{M}$. In picturesque language, $\tilde{M}$ consists of Minkowski space $M^{4}$, infinitely many "spheres of heaven" $Z^{n} M^{4}$ stacked above it and infinitely many "circles of hell" $Z^{-n} M^{4}$ below it $(n=1,2, \ldots)$. It carries the same conformal structure as the Einstein cosmos of general relativity [11].

In an exactly conformal invariant world there would be nothing to fix a Born radius, hence no atoms, no molecules and no physicists who could make observations. However we may resort to Gedanken experiments. The world line of a light signal, if there is such a thing in a conformal invariant world, could continue beyond our native Minkowski space to the distant past and future of superworld. Also, by an active conformal transformation an observer could be taken out of Minkowski space and put somewhere else on the superworld $\tilde{M}$.

Summing up our results briefly, we have shown that every Wightman field theory satisfying the hypothesis of weak conformal invariance is also globally invariant under the universal covering group $\left(\mathfrak{G}^{*}\right.$ of $\mathrm{SO}_{e}(4.2) / \mathbb{Z}_{2}$.

The central role in the proof of our results is played by a certain maximal noncommutative semigroup $\mathbb{S}$ contained in the Euclidean conformal group $\mathrm{SO}_{e}(5,1)$. Using positive definiteness of Euclidean Green functions à la Glaser [12] (a sharper version of Osterwalder Schrader axiom (E.2) which follows by using also locality [13]) and a newly derived conformal cluster property, it follows that $\checkmark$ acts as a semigroup of contractions on physical state space. We then make use of a generalized Hille-Yosida theorem for Lie-semigroups of contractions. Such

2 This depends on whether the boundary values of the analytically continued Wightman functions are distributions on $\tilde{M}$ - a difficult technical question which we have not investigated except for the two (and three-) point functions. 
a theorem is stated and proven in Appendix C. It should also be of considerable help in the general representation theory of noncompact groups. It asserts in particular that our contractive representation of the maximal semigroup $\mathbb{S}$ $C \mathrm{SO}_{e}(5,1)$ can be analytically continued to a unitary representation of the simply connected covering group $\mathfrak{5}^{*}$ of $\mathrm{SO}_{e}(4,2)$.

\section{Euclidean Green Functions}

Let us consider a local quantum field theory which satisfies the usual postulates (Wightman axioms): Temperedness, locality, spectrum condition, positivity, Lorentz invariance and uniqueness of the vacuum. Asymptotic completeness will however not be assumed since one knows that a nontrivial conformal invariant QFT is not a particle theory.

For simplicity we will consider a theory of one hermitean scalar field $\Phi(x)$. Generalization to fields of arbitrary spin will be discussed in Section 9.

Consider then the vector

$$
\Psi\left(x_{1} \ldots x_{n}\right)=\Phi\left(x_{1}\right) \ldots \Phi\left(x_{n}\right) \Omega, \quad \Omega=\text { vacuum } .
$$

This is a vector in the Hilbert space $\mathscr{H}$ of physical states after smearing with a test function. Because of the spectrum condition

$$
\Psi\left(x_{1} \ldots x_{n}\right)=\int d^{4} p d^{4} q_{1} \ldots d^{4} q_{n-1} \tilde{\Psi}\left(p, q_{1} \ldots q_{n-1}\right) \exp i\left\{p x_{1}+\Sigma q_{j}\left(x_{j+1}-x_{j}\right)\right\}
$$

with a $\tilde{\Psi}$ that vanishes unless $p^{0} \geqq 0$ and all $q_{i}^{0} \geqq 0(i=1 \ldots n-1)^{3}$. As a consequence, $\Psi$ can be analytically continued to a vector valued analytic function ${ }^{4} \Psi \in \mathscr{H}$, viz.

$$
\begin{aligned}
\Psi\left(z_{1} \ldots z_{n}\right) \quad \text { of } z_{k}=x_{k}+i y_{k} \quad & \text { defined and holomorphic for } \\
& y_{1} \in V_{+} \text {and } y_{j}-y_{i} \in V_{+} \text {if } j>i \\
& \left(V_{+}\right. \text {is the open forward light- } \\
& \text { cone })
\end{aligned}
$$

Glaser has pointed out (12) that locality and the edge of the wedge theorem can be used to further extend the domain of definition and analyticity:

$\Psi\left(z_{1} \ldots z_{n}\right)$ is defined as a vector in $\mathscr{H}$ and analytic in the $z_{k}$ in a connected domain which includes the Euclidean points with $z_{k}=\left(i y_{k}^{0}, \boldsymbol{x}_{k}\right)$ such that $y_{k}^{0}>0$ for all $k$ and $z_{i}^{0} \neq z_{j}^{0}$ for $i \neq j$.

Let us very briefly review the argument. Let $\pi$ any permutation of $(1 \ldots n)$ and consider the vector

$$
\Psi_{\pi}\left(z_{1} \ldots z_{n}\right)=\Psi\left(z_{\pi 1} \ldots z_{\pi n}\right), \quad z_{k}=x_{k}+i y .
$$

By the previous discussion this is defined and holomorphic in a domain containing the Euclidean points with $0<y_{\pi 1}^{0}<\ldots<y_{\pi n}^{0}$. Moreover, because of locality

$$
\begin{gathered}
\Psi_{\pi}\left(x_{1} \ldots x_{n}\right)=\Psi\left(x_{1} \ldots x_{n}\right) \text { for real } x_{k} \text { such that }\left(x_{i}-x_{j}\right)^{2}<0 \\
\text { for all } i \neq j,
\end{gathered}
$$

${ }^{3}$ Our metric in Minkowski space is $(+---)$.

4 We refer here to the notion of an analytic function with values in a normed space, $\mathrm{cp}$. Dieudonné [14], Chapter IX. 
i.e. for all the $\Psi_{\pi}$ agree on a real neighborhood. By the edge of the wedge theorem [3] they are therefore analytic continuations of one and the same analytic function. Its domain of analyticity must therefore contain the union (over all permutations $\pi$ ) of the previously established domains of analyticity of the individual $\Psi_{\pi}$, whence assertion (2.4).

To simplify the notation we will introduce Euclidean coordinates $\mathfrak{x}=\left(x^{4}, \boldsymbol{x}\right)$ which are real at Euclidean points, and we define

$$
\begin{gathered}
\Psi^{E}\left(\mathfrak{X}_{1} \ldots \mathfrak{X}_{n}\right)=\Psi\left(z_{1} \ldots z_{n}\right) \text { for } z=\left(i x_{k}^{4}, \boldsymbol{x}_{k}\right) \\
x_{k}^{4}>0, x_{i}^{4} \neq x_{j}^{4} \text { for all } k, i \neq j .
\end{gathered}
$$

The Wightman functions are defined by

$$
W_{n}\left(x_{1} \ldots x_{n}\right)=\left(\Omega, \Psi\left(x_{1} \ldots x_{n}\right)\right),
$$

and the Schwinger functions, also called Euclidean Green functions

$$
G_{n}\left(\mathfrak{x}_{1} \ldots \mathfrak{x}_{n}\right)=\left(\Omega, \Psi^{E}\left(\mathfrak{x}_{1} \ldots \mathfrak{x}_{n}\right)\right) .
$$

They are obtained from the Wightman functions by analytic continuation as is evident from our discussion. Because of translational and Lorentz invariance they are actually well defined and analytic for all $\mathfrak{x}_{k}$ such that $\mathfrak{x}_{i} \neq \mathfrak{x}_{j}$ for $i \neq j$ $[3,13]$.

It has been shown in [13] that the Euclidean Green functions are distributions sucht that

$$
G_{n}(f) \equiv \int d^{4} \mathfrak{x}_{1} \ldots d^{4} \mathfrak{x}_{n} f\left(\mathfrak{x}_{1} \ldots \mathfrak{x}_{n}\right) G_{n}\left(\mathfrak{x}_{1} \ldots \mathfrak{x}_{n}\right)
$$

is defined for all Schwartz test functions $f \in \mathscr{S}$ which vanish with all their derivatives at coinciding arguments.

We introduce the Euclidean time reversal operator $\theta$,

$$
\theta \mathfrak{x}=\left(-x^{4}, \boldsymbol{x}\right)
$$

so that

$$
z=\left(i x^{4}, \boldsymbol{x}\right) \text { implies } \bar{z}=\left(-i x^{4}, \boldsymbol{x}\right)=\left(i \theta x^{2}, \theta \boldsymbol{x}\right) .
$$

One finds from Eqs. (2.1) and (2.8) by uniqueness of analytic continuation that

$$
\left(\Psi^{E}\left(\mathfrak{x}_{1}, \ldots \mathfrak{x}_{m}\right), \Psi^{E}\left(\mathfrak{x}_{1}^{\prime}, \ldots, \mathfrak{x}_{n}^{\prime}\right)\right)=G_{m+n}\left(\theta \mathfrak{x}_{m}, \ldots, \theta \mathfrak{x}_{1}, \mathfrak{x}_{1}^{\prime}, \ldots, \mathfrak{x}_{n}^{\prime}\right) .
$$

Let us next introduce the space of test functions $\mathscr{S}_{+}^{0}$ which consists of Schwartz testfunctions $f\left(\mathfrak{x}_{1} \ldots \mathfrak{x}_{n}\right)$ which vanish with all their derivatives when $\mathfrak{x}_{i}=\mathfrak{x}_{j}$ for an $i \neq j$ or some $x_{i}^{4} \leqq 0$. For $f_{n}, h_{n} \in \mathscr{S}_{+}^{0}$ we define

$$
\left(\theta f_{n}^{*} \times h_{m}\right)\left(\mathfrak{x}_{1}, \ldots, \mathfrak{x}_{n}, \mathfrak{x}_{1}^{\prime} \ldots \mathfrak{X}_{m}^{\prime}\right)=\bar{f}_{m}\left(\theta \mathfrak{X}_{n}, \ldots, \theta \mathfrak{x}_{1}\right) h_{m}\left(\mathfrak{x}_{1}^{\prime}, \ldots, \mathfrak{x}_{m}^{\prime}\right) .
$$

Let now $f=\left(f_{k}\right)$ denote a finite sequence $f_{0}, f_{1}\left(\mathfrak{x}_{1}\right), f_{2}\left(\mathfrak{x}_{1} \mathfrak{x}_{2}\right), \ldots f_{N}\left(\mathfrak{x}_{1} \ldots \mathfrak{x}_{N}\right)$ such that all $f_{k} \in \mathscr{S}_{+}^{0}$ and in addition $f_{k}$ vanishes with all its derivatives for coinciding times $x_{i}^{4}=x_{j}^{4}$. Because of assertions (2.4), (2.9) and (2.11),

$$
\Psi(f) \equiv \sum_{k} \int d^{4} \mathfrak{x}_{1} \ldots d^{4} \mathfrak{x}_{k} f_{k}\left(\mathfrak{x}_{1}, \ldots, \mathfrak{x}_{k}\right) \Psi^{E}\left(\mathfrak{x}_{1}, \ldots, \mathfrak{x}_{k}\right)
$$

will be well defined elements of Hilbert space for such $f$, with scalar products

$$
(\Psi(f), \Psi(h))=\sum_{k, l} G_{k+l}\left(\theta f_{k}^{*} \times h_{l}\right) .
$$


Hence in particular their norm is positive, viz.

$$
\sum_{k, l} G_{k+l}\left(\theta f_{k}^{*} \times f_{l}\right) \geqq 0 .
$$

We will lastly argue that Euclidean positivity (2.14) will in fact hold for all finite sequences $f=\left(f_{k}\right), f_{k} \in \mathscr{S}_{+}^{0}$. Indeed, it follows from the lemma stated in Appendix D that the domain of real analyticity of $\Psi^{E}\left(x_{1} \ldots x_{n}\right)$ can be extended to all $n$-tuples of noncoinciding arguments with all $x_{i}^{4}>0$, and so $\Psi(f)$ is a vector in Hilbert space for any finite sequence $f=\left(f_{k}\right), f_{k} \in \mathscr{S}_{+}^{0}$.

Summary. There are vectors $\Psi^{E}\left(\mathfrak{x}_{1} \ldots \mathfrak{x}_{n}\right)$ defined and real analytic for $x_{i}^{4}>0$ and $\mathfrak{x}_{i} \neq \mathfrak{x}_{j}(i \neq j)$. They are symmetric in their arguments $\mathfrak{x}_{1} \ldots \mathfrak{x}_{n}$. The scalar product of two such vectors is given by Eq. (2.11), viz.

$$
\left(\Psi^{E}\left(\mathfrak{x}_{1}, \ldots, \mathfrak{x}_{m}\right), \Psi^{E}\left(\mathfrak{x}_{1}^{\prime}, \ldots, \mathfrak{x}_{n}^{\prime}\right)\right)=G_{m+n}\left(\theta \mathfrak{x}_{m} \ldots \theta \mathfrak{x}_{1}, \mathfrak{x}_{1}^{\prime} \ldots \mathfrak{x}_{n}^{\prime}\right) .
$$

As a consequence, positivity (2.14) holds for all finite sequences of testfunctions $f=\left(f_{k}\right), f_{0} \in \mathbb{C}, f_{k} \in \mathscr{S}_{+}^{0}(k=1,2, \ldots)$.

We remark that $\Psi^{E}\left(\mathfrak{X}_{1} \ldots \mathfrak{X}_{n}\right)$ is also a (regular) vector valued distribution on $\mathscr{S}_{+}^{0}\left(\mathbb{R}^{4 n}\right)$ and for arbitrary sequence $U_{n}, n=1,2, \ldots$ of open sets $U_{n} \subset \mathbb{R}_{+}^{4 n}$ $=\left\{\left(\mathfrak{X}_{1}, \ldots, \mathfrak{X}_{n}\right) \mid x_{i}^{4}>0\right\}$ finite linear combinations of vectors of the form

$$
\Psi(f)=\int d^{4} \mathfrak{x}_{1} \ldots d^{4} \mathfrak{x}_{n} f\left(\mathfrak{x}_{1}, \ldots, \mathfrak{x}_{n}\right) \Psi^{E}\left(\mathfrak{x}_{1}, \ldots, \mathfrak{x}_{n}\right), \quad f \in \mathscr{S}_{+}^{0}, \quad \operatorname{supp} f \subset U_{n}
$$

and the vacuum $\Omega$ form a dense subspace of the Hilbert space of physical states.

\section{Weak Conformal Invariance}

Consider points $\mathfrak{x}$ in Euclidean space $\mathbb{R}^{4}$. We will supplement them by one point of infinity $\mathfrak{x}=\infty$, the resulting space will be denoted by $\overline{\mathbb{R}}^{4}$. It becomes a compact topological space if we specify that the sets of points $x$ with $|x|>\varepsilon^{-1}$ form neighborhoods of $\mathfrak{x}=\infty$.

To bring out its structure as a differentiable manifold it is convenient to carry out a stereographic projection. We do this in two steps.

First let us introduce [15] projective coordinates $\xi^{A}, A=1 \ldots 6$. They form positive light like 6 -vectors $\xi \in C_{5,1}^{+}(\mathbb{R})$, viz. $\xi^{6}>0, \xi \cdot \xi \equiv g_{A B} \xi^{A} \xi^{B}=0, g_{A B}$ $=\operatorname{diag}(----,-+)$. They are related to Euclidean coordinates $\mathfrak{x}=\left(x^{\alpha}\right)$ by

$$
x^{\alpha}=\xi^{\alpha} / \kappa(\alpha=1 \ldots 4), \quad \kappa=\xi^{6}+\xi^{5} .
$$

The point $\mathfrak{x}=\infty$ corresponds to $\xi^{A}=\lambda(\overrightarrow{0},-11)$, and in general 6 -vectors $\lambda \xi$ correspond to the same point $\mathfrak{x}$ for all $\lambda>0$.

In particular there corresponds then to every $x$ one 6 -vector $\xi$ of the form $\xi=(\xi, 1)$ where $\xi \in S^{4}$ is a unit 5-vector [16], i.e. belongs to the sphere $S^{4}$. Explicitly

$$
\xi^{A}=\xi^{A} / \xi^{6}(A=1 \ldots 5), \quad \text { resp. } \quad \xi^{\alpha}=\kappa x^{\alpha}, 2 \boldsymbol{\kappa}^{-1}=1+|\mathfrak{x}|^{2} .
$$

Thus $\xi$ and $x$ are related by a stereographic projection (s. Fig. 1) and the neighborhoods of $\mathfrak{x}=\infty$ correspond to neighborhoods of $\xi_{\infty}=(\overrightarrow{0},-1)$. Thus $\overline{\mathbb{R}}^{4}$ and $S^{4}$ are homeomorphic, and $S^{4}$ is of course a differentiable manifold. Most important is the fact that they are compact. 


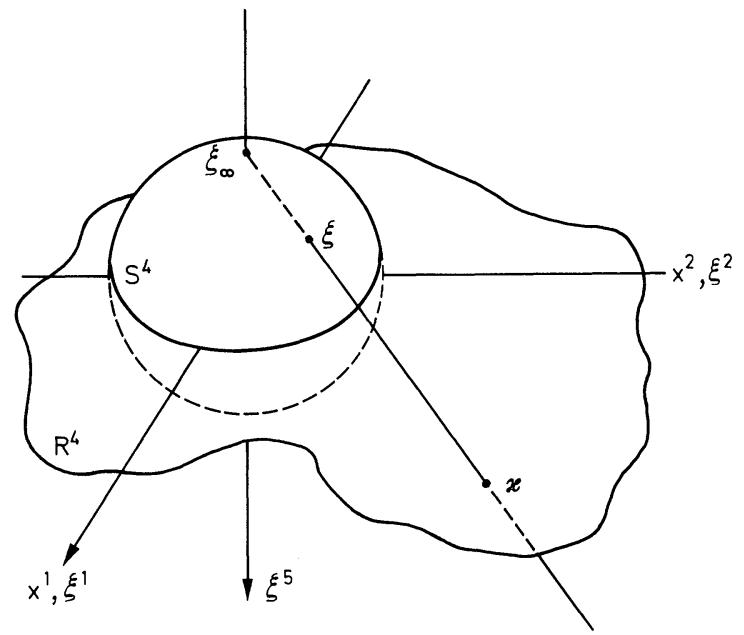

Fig. 1. Stereographic Projection of $\xi \in S^{4}$ onto $x \in \mathbb{R}^{4}$. Drawing for 2 space dimensions

Conformal transformations $\Lambda \in \mathrm{SO}_{e}(5,1)$ are $6 \times 6$ matrices $\Lambda_{B}^{A}$ satisfying the conditions $\Lambda^{K}{ }_{M} g_{K L} \Lambda^{L}{ }_{M}=g_{M N}$, $\operatorname{det} \Lambda=1$ and $\Lambda_{6}^{6} \geqq 1$. In a neighborhood of the identity we may expand

$$
\Lambda=1-\frac{i}{2} \omega^{A B} J_{A B}+\ldots \quad \omega^{A B}=-\omega^{B A} \quad \text { real. }
$$

The generators $J_{A B}$ are $6 \times 6$-matrices satisfying commutation relations [6]

$$
\left[J_{K L}, J_{M N}\right]=i\left(g_{K N} J_{L M}+g_{L M} J_{K N}-g_{K M} J_{L N}-g_{L N} J_{K M}\right) .
$$

$J_{A B}$ generates an infinitesimal pseudorotation in the $A B$-plane.

Conformal transformations $\Lambda$ act as pseudo-rotations on 6-vectors $\xi$,

$$
\xi \rightarrow \xi^{\prime}=\Lambda \xi, \quad \text { viz. } \quad \xi^{A}=\Lambda^{A}{ }_{B} \xi^{B},
$$

The Euclidean time reversal $\theta$ will also be used. It acts as

$$
\xi \rightarrow \theta \xi=\left(\xi^{1} \ldots \xi^{3},-\xi^{4}, \xi^{5} \xi^{6}\right) .
$$

Since $\Lambda$ commutes with similarity transformations $\xi \rightarrow \lambda \xi, \lambda>0$, this also induces a transformation of points $x$ of Euclidean space $\overline{\mathbb{R}}^{4}$. The explicit expressions for these can be found in [6] (4-vectors should be read as Euclidean ones), we will simply write it as $\mathfrak{x} \rightarrow \mathfrak{x}^{\prime}=\Lambda \mathfrak{x}$. Similarly, (3.4) induces a transformation on the unit 5 -vectors $\xi$ defined by (3.2); they are diffeomorphisms of $S^{4}$ [16].

One also defines a measure on the cone $C_{5,1}^{+}$by

$$
d \mu(\xi)=2 d^{6} \xi \theta\left(\xi^{6}\right) \delta(\xi \cdot \xi) \delta(\xi \cdot \eta-1)
$$

where $\eta$ is an arbitrary positive timelike or lightlike 6-vector.

In the following discussion, $d$ will denote the dimension of the field $\Phi(x)$, it can be read off from the two-point function as explained e.g. in [15]. 
The state vectors $\Psi^{E}$ and Euclidean Green functions will be defined as functions of the projective coordinates by

$$
\Psi^{E}\left(\xi_{1} \ldots \xi_{n}\right)=\kappa_{1}^{-d} \ldots \kappa_{n}^{-d} \Psi^{E}\left(\mathfrak{x}_{1} \ldots \mathfrak{x}_{n}\right) \quad \text { resp. } \quad G_{n}\left(\xi_{1} \ldots \xi_{n}\right)=\kappa_{1}^{-d} \ldots \kappa_{n}^{-d} G_{n}\left(\mathfrak{x}_{1} \ldots x_{n}\right)
$$

they are homogeneous of degree $-d$ in each variable separately.

Similarly we write for test functions $f$

$$
f\left(\xi_{1} \ldots \xi_{n}\right)=\kappa_{1}^{d-4} \ldots \kappa_{n}^{d-4} f\left(\mathfrak{x}_{1} \ldots \mathfrak{x}_{n}\right),
$$

$G_{n}(f)$ as defined by Eq. (2.9) may then also be written as [15]

$$
G_{n}(f)=\int d \mu\left(\xi_{1}\right) \ldots d \mu\left(\xi_{n}\right) f\left(\xi_{1} \ldots \xi_{n}\right) G_{n}\left(\xi_{1} \ldots \xi_{n}\right) .
$$

This is evident if $\eta^{A}=(\overrightarrow{0}, 11)$ is chosen in the measure (3.5), and holds then generally because the integral is independent of $\eta$ by the basic covariance lemma (s. Appendix A). Note that by chosing $\eta=(\overrightarrow{0}, 01)$ the integration can be made to run over the sphere $S^{4}$ of variables $\xi=(\xi, 1)$. We will for this purpose abbreviate

$$
f\left(\xi_{1} \ldots \xi_{n}\right) \equiv f\left(\left(\xi_{1}, 1\right) \ldots\left(\xi_{n}, 1\right)\right) .
$$

Finally we define an action of conformal transformations on test functions $f$ by

$$
(\Lambda f)\left(\xi_{1} \ldots \xi_{n}\right)=f\left(\Lambda^{-1} \xi_{1} \ldots \Lambda^{-1} \xi_{n}\right) \text {. }
$$

This induces a transformation on test functions $f\left(x_{1} \ldots x_{n}\right)$, the explicit formulae for them can be found e.g. in [1].

The hypothesis of weak conformal invariance says that the Euclidean Green functions should be invariant under conformal transformations $\Lambda \in \mathrm{SO}_{e}(5,1)$. That is

$$
G_{n}(\Lambda f)=G_{n}(f) \text { for all } \Lambda \in \mathrm{SO}_{e}(5,1)
$$

and test functions $f$ such that the right hand side (r.h.s.) exists; hence in particular for $f \in \mathscr{S}_{+}^{0}$.

In the forthcoming sections we will make extensive use of the test function space $\mathscr{S}_{+}^{0}$. Recall its definition:

$$
\begin{aligned}
\mathscr{S}_{+}^{0} \equiv\left\{f \in \mathscr{S} \mid f\left(\mathfrak{x}_{1} \ldots \mathfrak{x}_{n}\right)\right. \text { vanishes with all its derivatives } \\
\text { if } \left.\mathfrak{x}_{i}=\mathfrak{x}_{j}\left(\text { some } i \neq j \text { ) or } x_{i}^{4} \leqq 0 \text { (some } i\right)\right\} .
\end{aligned}
$$

$\mathscr{S}$ is the space of Schwartz test functions [3]. The topology on $\mathscr{S}\left(\mathbb{R}^{n}\right)$ is given by a countable family of norms, viz.

$$
|f|_{m} \equiv \max _{|\alpha| \leqq m} \sup _{x \in \mathbf{R}^{n}}\left(1+|x|^{2}\right)^{m / 2}\left|f^{(\alpha)}(x)\right| ; \quad m=0,1,2 \ldots
$$

$\alpha \equiv\left(\alpha_{1} \ldots \alpha_{n}\right)$ is a multiindex and $f^{(\alpha)}(x) \equiv \frac{\partial^{\alpha_{1}}}{\partial x_{1}^{\alpha_{1}}} \ldots \frac{\partial^{\alpha_{n}}}{\partial x_{n}^{\alpha_{n}}} f(x) \quad|\alpha| \equiv \sum_{i=1}^{n} \alpha_{i}$; $|x|^{2} \equiv \sum_{i=1}^{n}\left(x_{i}\right)^{2}$.

The only topology on $\mathscr{S}_{+}^{0}$ used will be that inherited from $\mathscr{S}$, i.e. it is defined by the norms (3.13). The vectors $\Psi^{E}\left(x_{1} \ldots x_{n}\right)$ are then vector valued distributions on $\mathscr{S}_{+}^{0}$, which means that

$$
\|\Psi(f)\| \rightarrow 0 \quad \text { if } \quad f \rightarrow 0\left(\text { in } \mathscr{S}_{+}^{0}\right) .
$$


Formulae (3.7) and (3.9) tell us finally that the elements of $\mathscr{S}_{+}^{0}$ may be viewed as functions $f\left(\xi_{1} \ldots \xi_{n}\right)$ defined on $S^{4} \times \cdots \times S^{4}$. They are infinitely differentiable and vanish with all their derivatives for coinciding arguments and if some $\xi_{i}^{4} \leqq 0$. Furthermore $f \rightarrow 0$ (in $\mathscr{S}_{+}^{0}$ ) iff $f\left(\xi_{1} \ldots \xi_{n}\right) \rightarrow 0$ together with all its derivatives uniformly on some neighborhood of every point of the compact manifold $S^{4} \mathfrak{x} \cdots \mathfrak{x} S^{4}$.

\section{A Semigroup}

Let $\mathfrak{6}$ the Euclidean conformal group which acts on Euclidean coordinates $\mathfrak{x}$ as explained in Section 3 .

Let $\mathfrak{S}$ the set of elements of $\mathfrak{G}$ which leaves invariant the halfspace $x^{4}>0$, $\mathfrak{x} \neq \infty$. In other words, for every $\Lambda \in \mathfrak{S}, x^{4}>0, \mathfrak{x} \neq \infty$ implies $(\Lambda \mathfrak{x})^{4}>0$ and $\Lambda$ does not take any such point to infinity. Evidently $\Lambda_{1} \Lambda_{2} \in \Im$ if $\Lambda_{1}, \Lambda_{2} \in \mathbb{S}$, thus $\Im$ is a semigroup.

To determine $\subseteq$ explicitly, we introduce hyperbolic coordinates ${ }^{5}$. Let $\xi$ the projective coordinates of Section 3, viz. $\xi^{\alpha}=\kappa x^{\alpha}, \kappa=\xi^{6}+\xi^{5}$. Put

$$
\xi^{6}=r \operatorname{ch} \sigma, \xi^{4}=r \operatorname{sh} \sigma, \xi^{k}=r e^{k}(k=123,5), \quad r>0
$$

with $\left(e^{k}\right)$ a unit 4 -vector. We see that $x^{4}>0, \mathfrak{x} \neq \infty$ if and only if $\xi^{4}>0$ and this is true if and only if $\sigma>0$. Here and everywhere, $>$ means strictly larger than", otherwise we write $\geqq$.

We introduce the subgroup $\mathfrak{U} \simeq \mathrm{SO}_{e}(4,1)$ which consist of pseudo-rotations that leave invariant the 4 -th coordinate $\xi^{4}$. It is generated by $J_{A B}$ with $A, B \neq 4$. Let further

$$
H=-i J_{64} \quad \text { and } \quad b_{\tau} \equiv e^{-H \tau}
$$

$b_{\tau}$ is of course an element of $\mathfrak{6}$. Explicitly, if $\xi$ is given by (4.1) then

has components

$$
\xi^{\prime}=b_{\tau} \xi
$$

$$
\xi^{\prime 6}=r \operatorname{ch}(\sigma+\tau), \quad \xi^{\prime 4}=r \operatorname{sh}(\sigma+\tau) ; \quad \xi^{\prime k}=r e^{k} \quad(k=123,5) .
$$

Consider now the set $\mathfrak{\Im}^{0}$ of group elements $\Lambda$ of the form:

$$
\mathfrak{\Im}^{0}: \Lambda=u_{1} e^{-\boldsymbol{H} \tau} u_{2}=u_{1} b_{\tau} u_{2} \quad \text { with } \quad \tau>0 \quad \text { and } \quad u_{i} \in \mathfrak{U} \quad(i=1,2) \text {. }
$$

Evidently $\mathfrak{\Im}^{0}$ is contained in $\mathfrak{\Xi}$. Indeed $\mathfrak{U} \subset \mathfrak{\Xi}$ since $u \in \mathfrak{U}$ does not affect $\xi^{4}$ and therefore leaves invariant the halfspace $\xi^{4}>0$. Also $b_{\tau} \in \Xi$ for $\tau>0$ since it translates the variable $\sigma \rightarrow \sigma+\tau$ by a positive amount.

Toller and collaborators have shown ${ }^{6}[17]$ that the closure of $\mathfrak{S}^{0}$ is a maximal semigroup contained in $\mathfrak{G}$, i.e. there is no semigroup containing it properly except $6 \mathfrak{6}$. It follows that every element in the interior of $\mathfrak{S}$ can be parametrized in the form (4.4). In fact $\mathfrak{S}^{0}$ is just the interior of $\mathfrak{\subseteq}$ and is itself a semigroup [17].

It is interesting to observe that under time reversal

$$
\bar{\Lambda} \equiv \theta \Lambda^{-1} \theta \in \mathbb{S}^{\sim} \text { for } \Lambda \in \mathbb{S}^{\sim} \equiv \mathbb{S}^{0} \cup \mathfrak{U} \text {. }
$$

5 They are singular at the two points $\xi^{6}= \pm \xi^{4}, \xi^{k}=0(k=123,5)$. These correspond to the points $\sigma= \pm \infty, r=0, e$ irrelevant.

6 Their discussion is for $\mathrm{SO}_{e}(3,1)$ but carries over immediately. 
This is evident from the definition of $\mathfrak{\Xi}$. In particular

$$
\theta H \theta=-H \text { and so } \theta b_{\tau}^{-1} \theta=b_{\tau}=b_{-\tau}^{-1} .
$$

We will later on need some other special transformations contained in $\Xi$. It is easiest to specify their action in $\mathfrak{x}$-space:

$$
\begin{array}{ll}
\text { dilatations: } & d_{\varrho}: \mathfrak{x} \rightarrow \varrho \mathfrak{x}, \varrho>0 . \\
\text { time translations by } a>0: & t_{a}: \quad \mathfrak{x} \rightarrow \mathfrak{x}+\mathfrak{a}, \quad \mathfrak{a}=(\mathbf{0}, a) .
\end{array}
$$

Dilatations are generated by $J_{56}$ and are therefore in $\mathfrak{U}$, while $t_{a}$ turns out to be in $\mathfrak{S}$ but not in its interior $\mathbb{S}^{0}$.

The action of elements $\Lambda$ of $\mathfrak{S}$ on test functions $f$ is defined by Eq. (3.10), which applies for all $\Lambda \in \mathfrak{G}$. We will now proceed to state three lemmas about elements of the semigroup $\subseteq$ and their action on test functions $f\left(x_{1} \ldots \mathfrak{x}_{n}\right)$ :

a) $\mathfrak{S}$ leaves invariant the test function space $\mathscr{S}_{+}^{0}$, i.e. if $f \in \mathscr{S}_{+}^{0}$ then $\Lambda f \in \mathscr{S}_{+}^{0}$ for all $\Lambda \in \mathbb{\Xi}$.

b) Define $\Lambda_{\tau}$ by

$$
b_{\tau}=d_{\mathrm{ch} \tau}^{-1} t_{\mathrm{sh} \tau} \Lambda_{\tau} .
$$

Then $\Lambda_{\tau}$ tends to a limit, $\Lambda_{\tau} \rightarrow \Lambda_{\infty} \in \mathbb{G}$ as $\tau \rightarrow \infty$, and $\Lambda_{\infty}$ takes the point $\mathfrak{x}=(-1, \mathbf{0})$ to $\mathfrak{x}^{\prime} \equiv \Lambda_{\infty} \mathfrak{x}=\infty$.

c) If $f \in \mathscr{S}_{+}^{0}$ then $t_{a} \Lambda_{\tau} f \in \mathscr{S}_{+}^{0}$ for $2 \leqq \tau \leqq \infty$ and $2 \leqq a$.

Proof. a) It is most convenient to use the characterization of $\mathscr{S}_{+}^{0}$ as a space of functions $f\left(\xi_{1} \ldots \xi_{n}\right)$, see Section 3. Since $\Lambda \in \mathbb{S}$ leaves invariant the halfspace $\xi^{4}>0$ and of course also the sets of coinciding points $\xi_{i}=\xi_{j}$, the support properties etc. of functions $f \in \mathscr{S}_{+}^{0}$ are left invariant. Moreover, $\Lambda$ are analytic mappings of the sphere $S^{4}$ (a homogeneous space of (5) [18], therefore they map infinitely differentiable functions into infinitely differentiable functions.

b) This has to be verified by explicit computation with $6 \times 6$ metrices, the computation will be done in Appendix B.

c) Functions $f \in \mathscr{S}_{+}^{0}$ vanish by definition if $x_{i}^{4} \leqq 0$ for some $i$. Hence

$$
\text { supp } \Lambda_{\tau} f=\Lambda_{\tau} \operatorname{supp} f \subset \Lambda_{\tau}\left\{\left(\mathfrak{x}_{1} \ldots \mathfrak{x}_{n}\right) ; \quad x_{i}^{4} \geqq 0 \quad \text { for all } i\right\} .
$$

As can be seen from the explicit form of the matrices $\Lambda_{\tau}$ (see Appendix B), $\Lambda_{\tau}\left\{\left(\mathfrak{x}_{1} \ldots \mathfrak{x}_{n}\right) ; x_{i}^{4} \geqq 0(\forall i)\right\}$ is contained in $\left\{\left(\mathfrak{x}_{1} \ldots \mathfrak{x}_{n}\right) ;\left|x_{i}\right|^{2} \leqq 2(\forall i)\right\}$ whenever $\tau \geqq 2$. This proves our assertion.

\section{Conformal Cluster Property}

We will now state and prove a cluster property of Euclidean Green functions which holds in every weakly conformal invariant Wightman QFT with a unique vacuum. It reads as follows:

$$
G_{m+n}\left(\theta f^{*} \times b_{\tau} g\right) \rightarrow G_{m}\left(\theta f^{*}\right) G_{n}(g) \quad \text { for } \quad \tau \rightarrow+\infty
$$

and

$$
f\left(\mathfrak{x}_{1} \ldots \mathfrak{x}_{m}\right), \quad g\left(\mathfrak{x}_{1}^{\prime} \ldots \mathfrak{x}_{n}^{\prime}\right) \in \mathscr{S}_{+}^{0} .
$$


$b_{\tau}$ is the translation of the hyperbolic coordinate $\sigma$ defined in the last section; for other notation see Section 2.

To prove cluster property (5.1) we start from the conventional' cluster property of Euclidean Green functions in the time-direction, viz.

and

$$
G_{m+n}\left(\theta f^{*} \times t_{a} g\right) \rightarrow G_{m}\left(\theta f^{*}\right) G_{n}(g) \text { for } a \rightarrow+\infty
$$

$$
f, g \in \mathscr{S}_{+}^{0} .
$$

Assertion (5.2) is proven by the following argument. By the spectrum condition, energy operator $P^{0} \geqq 0$ and the vacuum $\Omega$ is by assumption its only eigenvector with eigenvalues 0 . Therefore

$$
T\left(t_{a}\right) \equiv \exp \left(-P^{0} a\right) \rightarrow E_{\Omega} \quad \text { (weakly) as } a \rightarrow+\infty
$$

with $E_{\Omega}$ the projection operator on the vacuum. From the work of Osterwalder and Schrader [13] we obtain the action of $t_{a}$ on vectors $\Psi(f)$ defined in Section 2 as

$$
\left(\Psi(f), T\left(t_{a}\right) \Psi(g)\right)=\left(\Psi(f), \Psi\left(t_{a} g\right)\right)
$$

with $t_{a} g$ defined by Eqs. (4.5), (3.10).

Inserting Eqs. (2.13) and (2.8b) we obtain then from (5.3)

$$
G_{m+n}\left(\theta f^{*} \times t_{a} g\right) \rightarrow\left(\Psi(f), E_{\Omega} \Psi(g)\right)=G_{m}\left(\theta f^{*}\right) G_{n}(g)
$$

as $a \rightarrow+\infty$. q.e.d.

Consider now the left hand side of (5.1). Writing $2 \tau$ in place of $\tau$ we have

$$
G_{m+n}\left(\theta f^{*} \times b_{2 \tau} g\right)=G_{m+n}\left(\theta b_{\tau} f^{*} \times b_{\tau} g\right)
$$

by (4.4b) and weak conformal invariance (3.11). This is

$$
=G_{m+n}\left(\theta d_{\mathrm{ch} \tau}^{-1} t_{\mathrm{sh} \tau} f_{\tau}^{*} \times d_{\mathrm{ch} \tau}^{-1} t_{\mathrm{sh} \tau} g_{\tau}\right) \quad \text { with } \quad f_{\tau} \equiv \Lambda_{\tau} f, \quad g_{\tau} \equiv \Lambda_{\tau} g, \quad(\tau \leqq \infty)
$$

$\Lambda_{\tau}$ as defined in (4.6b). Since $\theta d_{\mathrm{ch} \tau}^{-1} t_{\operatorname{sh} \tau}=d_{\mathrm{ch} \tau}^{-1} t_{-\operatorname{sh} \tau} \theta$ by (4.5) it follows by using conformal invariance again that this is

$$
=G_{m+n}\left(\theta f_{\tau}^{*} \times t_{2 \operatorname{sh} \tau} g_{\tau}\right) .
$$

Because of the conventional cluster property (5.2)

$$
G_{m+n}\left(\theta f_{\infty}^{*} \times t_{2 \mathrm{sh} \tau} g_{\infty}\right) \rightarrow G_{m}\left(\theta f_{\infty}^{*}\right) G_{n}\left(g_{\infty}\right)=G_{m}\left(\theta f^{*}\right) G(g) .
$$

We are therefore left with the problem of showing that

$$
R \equiv G_{m+n}\left(\theta f_{\infty}^{*} \times t_{2 \operatorname{sh} \tau} g_{\infty}\right)-G_{m}\left(\theta f_{\tau}^{*} \times t_{2 \mathrm{sh} \tau} g_{\tau}\right) \rightarrow 0 \text { as } \tau \rightarrow+\infty .
$$

Again we shift an operator $t_{\mathrm{sh} \tau}$ to the left, obtaining

$$
R=G_{m+n}\left(\theta t_{\mathrm{sh} \tau} f_{\infty}^{*} \times t_{\mathrm{sh} \tau} g_{\infty}\right)-G_{m+n}\left(\theta t_{\mathrm{sh} \tau} f_{\tau}^{*} \times t_{\mathrm{sh} \tau} g_{\tau}\right) .
$$

By (4.6), $t_{\mathrm{sh} \tau} f_{\infty}, t_{\mathrm{sh} \tau} g_{\infty}, t_{\mathrm{sh} \tau} f_{\tau}$ and $t_{\mathrm{sh} \tau} g_{\tau}$ are elements of $\mathscr{S}_{+}^{0}$ for sufficiently large $\tau$, say $\tau \geqq \varrho$. For such $\tau$ we may form the vectors $\Psi\left(t_{\mathrm{sh} \tau} f_{\infty}\right)$, etc. and obtain from (2.13)

$$
\begin{aligned}
R= & \left(\Psi\left(t_{\mathrm{sh} \tau} f_{\infty}\right), \Psi\left(t_{\mathrm{sh} \tau} g_{\infty}\right)\right)-\left(\Psi\left(t_{\mathrm{sh} \tau} f_{\tau}\right), \Psi\left(t_{\mathrm{sh} \tau} g_{\tau}\right)\right) \\
= & \left(\Psi\left(t_{\mathrm{sh} \tau} f_{\infty}\right)-\Psi\left(t_{\mathrm{sh} \tau} f_{\tau}\right), \Psi\left(t_{\mathrm{sh} \tau} g_{\infty}\right)\right) \\
& +\left(\Psi\left(t_{\mathrm{sh} \tau} f_{\tau}\right), \Psi\left(t_{\mathrm{sh} \tau} g_{\infty}\right)-\Psi\left(t_{\mathrm{sh} \tau} g_{\tau}\right)\right) .
\end{aligned}
$$


Now, $\Psi\left(t_{a} f\right)=T\left(t_{a}\right) \Psi(f)$, and since $P^{0} \geqq 0, T\left(t_{a}\right)$ is contractive, viz. $\left\|T\left(t_{a}\right)\right\| \leqq 1$. Therefore

$$
\begin{aligned}
|R| \leqq & \left\|\Psi\left(t_{\text {sh } \varrho} g_{\infty}\right)\right\|\left\|\Psi\left(t_{\text {sh } \varrho}\left(f_{\infty}-f_{\tau}\right)\right)\right\| \\
& +\left\|\Psi\left(t_{\text {sh } \varrho} f_{\tau}\right)\right\|\left\|\Psi\left(t_{\text {sh } \varrho}\left(g_{\infty}-g_{\tau}\right)\right)\right\| \rightarrow 0 \text { as } \tau \rightarrow \infty
\end{aligned}
$$

because $\Psi(\cdot)$ is a vector valued distribution, and $t_{\text {sh } \varrho} f_{\tau} \rightarrow t_{\text {sh } \varrho} f_{\infty}, t_{\text {sh } \varrho} g_{\tau} \rightarrow t_{\text {sh } \varrho} g_{\infty}$ in the topology of $\mathscr{S}_{+}^{0}(\tau \rightarrow \infty)$.

\section{Unitary Representation of the Quantum Mechanical Conformal Group}

Consider Euclidean conformal transformations $\Lambda$ in the semigroup $\mathbb{\Xi}$. We define their action on state vectors $\Psi(f)$ by

$$
T(\Lambda) \Psi(f)=\Psi(\Lambda f), \quad \Lambda \in \mathbb{S} .
$$

For $f=\left(f_{k}\right)$ a finite sequence of testfunctions $f_{k} \in \mathscr{S}_{+}^{0}, \Lambda f=\left(\Lambda f_{k}\right)$. By Lemma (4.6a), $\Lambda f_{k}$ will be in $\mathscr{S}_{+}^{0}$ again for every $\Lambda \in \mathcal{G}$, and the r.h.s. of Eq. (6.1) is thus well defined. Now it may happen that $\Psi(f)=0$ for some $f \neq 0$. To show that Definition (6.1) is meaningful nevertheless it must be demonstrated that $\Psi(f)=0$ implies $\Psi(\Lambda f)=0$ for all $\Lambda \in \mathbb{S}$, i.e. the kernel of the homomorphism $f \rightarrow \Psi(f)$ is invariant under the semigroup. We will come to this in a moment.

Define $\bar{\Lambda}=\theta \Lambda^{-1} \theta$ as in (4.4a). It follows then from (6.1) that

$$
(\Psi(g), T(\Lambda) \Psi(f))=(T(\bar{\Lambda}) \Psi(g), \Psi(f))
$$

Indeed, by (6.1) and expression (2.13) for the scalar product, the left hand side (l.h.s.) is

$$
\Sigma G_{k+l}\left(\theta g_{k}^{*} \times \Lambda f_{l}\right)=\Sigma G_{k+l}\left(\Lambda^{-1} \theta g_{k}^{*} \times f_{l}\right)=\Sigma G_{k+l}\left(\theta \Lambda g_{k}^{*} \times f_{l}\right)=\text { r.h.s. }
$$

We used weak conformal invariance (3.11) in the first equality.

The same consideration also shows that $\Psi(f)=0$ implies $\Psi(\Lambda f)=0$ as is required for consistency. Indeed, $\Psi(f)=0$ if and only if $(\Psi(g), \Psi(f))=0$ for all finite sequences $g=\left(g_{k}\right), g_{k} \in \mathscr{S}_{+}^{0}$. But then $(\Psi(g), \Psi(\Lambda f))=(\Psi(\bar{\Lambda} g), \Psi(f))=0$ for all $g$, hence $\Psi(\Lambda f)=0$.

Let us now restrict our attention to elements in $\mathfrak{\Im}^{\sim}=\mathfrak{\Im}^{0} \cup \mathfrak{U}, \mathfrak{S}^{0}$ the interior of $\mathfrak{S}$. $\mathbb{S}$ is also a semigroup. Every such element can be factorized as $\Lambda=u_{1} b_{\tau} u_{2}$, $\tau \geqq 0, u_{i} \in \mathfrak{U}$, see Section 4 . Consider the individual factors. Every $u \in \mathfrak{U}$ commutes with $\theta$ because it does not affect the 4-th coordinate of 6 -vectors $\xi$, therefore $\bar{u}=u^{-1}$. Equation $(6.2)$ reads then

$$
\left.(\Psi(g), T(u) \Psi(f))=\left(T\left(u^{-1}\right) \Psi(g), \Psi f\right)\right) .
$$

The vectors $\Psi(f)$ are dense in the Hilbert space $\mathscr{H}$ or physical states. This follows from the result of Osterwalder and Schrader that the same is still true of the seemingly smaller subspace of vectors $\Psi(f), f \subset \mathscr{S}_{<}^{0}$ in the notation of [13]. Therefore (6.3) extends by continuity to the whole Hilbert space and we have a unitary representation of the subgroup $\mathfrak{U}$ of $\mathfrak{\Xi}$ on $\mathscr{H}$. 
Consider next the boosts $b_{\tau}=\exp (-H \tau)$ in the $4-6$ plane, with $\tau \geqq 0$. By Eq. (4.4) we have $\bar{b}_{\tau}=b_{\tau}$ and therefore

$$
\left(\Psi(g), T\left(b_{\tau}\right) \Psi(f)\right)=\left(T\left(b_{\tau}\right) \Psi(g), \Psi(f)\right),
$$

i.e. $T\left(b_{\tau}\right)$ is hermitean. By its definition $b_{\tau} b_{\sigma}=b_{\tau+\sigma}$ and therefore by (6.1) also $T\left(b_{\tau}\right) T\left(b_{\sigma}\right)=T\left(b_{\tau} b_{\sigma}\right)(\sigma, \tau \geqq 0)$, i.e. they form a representation of a 1-parameter semigroup.

Next we will show that

$$
\left(\Psi(f), T\left(b_{\tau}\right) \Psi(f)\right) \leqq(\Psi(f), \Psi(f))=\|\Psi(f)\|^{2} \quad \text { for } \quad \tau \geqq 0 .
$$

Proof. This inequality follows from the conformal cluster property (5.1). Written in terms of state vectors it reads

$$
\begin{gathered}
\left(\Psi(g), T\left(b_{\tau}\right) \Psi(f)\right) \rightarrow(\Psi(g), \Omega)(\Omega, \Psi(f)) \quad \text { for } \quad \tau \rightarrow+\infty \\
f=\left(f_{k}\right) ; \quad g=\left(g_{l}\right) ; \quad f_{k}, g_{l} \in \mathscr{S}_{+}^{0} .
\end{gathered}
$$

Without loss of generality we may assume $\|\Psi(f)\|=1$. By the Cauchy Schwarz inequality we have then

$$
\left(\Psi(f), T\left(b_{\tau}\right) \Psi(f)\right) \leqq\left(T\left(b_{\tau}\right) \Psi(f), T\left(b_{\tau}\right) \Psi(f)\right)^{1 / 2}=\left(\Psi(f), T\left(b_{2 \tau}\right) \Psi(f)\right)^{1 / 2} .
$$

The last equality follows from hermiticity (6.4). Repeating the argument $n$ times we get

$$
\left(\Psi(f), T\left(b_{\tau}\right) \Psi(f)\right) \leqq\left(\Psi(f), T\left(b_{2^{n_{\tau}}}\right) \Psi(f)\right)^{2-n} \rightarrow c \leqq 1 \quad \text { as } \quad n \rightarrow \infty .
$$

In the last step we used cluster property (6.8), noting that $|(\Omega, \Psi(f))| \leqq\|\Psi(f)\|^{2}$. This proves inequality (6.5) for all finite sequences $f=\left(f_{k}\right), f_{k} \in \mathscr{S}_{+}^{0}$.

Inequality (6.5) can be extended by continuity to all of Hilbert space $\mathscr{H}$. It means that $\left\|T\left(b_{\tau}\right)\right\| \leqq 1$ for all $\tau \geqq 0$. Now every element $\Lambda$ of $\Xi^{\sim}$ may be written in the form $\Lambda=u_{1} b_{\tau} u_{2} ; u_{i} \in \mathfrak{U}, \tau \geqq 0$. Therefore by the representation property $T(\Lambda)=T\left(u_{1}\right) T\left(b_{\tau}\right) T\left(u_{2}\right)$. Since $T\left(u_{i}\right)$ are unitary, it follows that

$$
\|T(\Lambda)\| \leqq 1 \text { for all } \Lambda \in \mathfrak{S}^{\sim}=\mathfrak{S}^{0} \cup \mathfrak{H} .
$$

That is, we can extend the representation of $\widetilde{\varsigma}$ defined by (6.1) to all of Hilbert space $\mathscr{H}$ by continuity, and we obtain in this way a representation of $\Xi^{\sim}$ by contraction operators on $\mathscr{H}$. By (6.2) it satisfies

$$
(\Psi, T(\Lambda) \Phi)=(T(\bar{\Lambda}) \Psi, \Phi) \quad \text { for } \quad \Lambda \in \Xi^{\sim}, \quad \bar{\Lambda}=\theta \Lambda^{-1} \theta ; \quad \Psi, \Phi \in \mathscr{H} .
$$

Lastly we will examine the continuity properties of this representation. The identity 1 of $\mathfrak{b}$ evidently belongs to $\mathfrak{S}^{\sim}$. Let then $\Lambda \rightarrow 1$ through values in $\mathfrak{S}^{\sim}$. It follows from definition (6.1) that

$$
(\Psi, T(\Lambda) \Phi) \rightarrow(\Psi, \Phi) \text { for } \Lambda \rightarrow 1,
$$

and state vectors of the form $\Psi=\Psi(f), \Phi=\Psi(g)$. Since $\|T(\Lambda)\| \leqq 1$, it follows that (6.10) holds for all $\Psi, \Phi$ in $\mathscr{H}$. But then also

$$
\|T(\Lambda) \Psi-\Psi\| \rightarrow 0 \quad \text { as } \quad \Lambda \rightarrow 1 .
$$


For we have

$$
\begin{aligned}
\|T(\Lambda) \Psi-\Psi\|^{2} & =(T(\Lambda) \Psi, T(\Lambda) \Psi)-(T(\Lambda) \Psi, \Psi)-(\Psi, T(\Lambda) \Psi)+(\Psi, \Psi) \\
& =([T(\bar{\Lambda} \Lambda)-T(\Lambda)-T(\bar{\Lambda})+1] \Psi, \Psi) \rightarrow 0 \quad \text { if } \quad \Lambda \rightarrow 1
\end{aligned}
$$

by (6.11), since $\Lambda \rightarrow 1$ implies $\bar{\Lambda} \rightarrow 1$.

Summing up we have a representation of $\mathfrak{S}$ by contraction operators $T(\Lambda)$ on Hilbert space $\mathscr{H}$ which satisfies relation $(6.10)$ and is strongly continuous at the identity in the sense that (6.12) holds.

We will now apply the generalized Hille Yosida theorem which is stated and proven in Appendix C. To show that its hypothesis are satisfied it only remains to be shown that the semigroup $\mathfrak{S}^{0}$ considered so far coincides with that defined in (C.1). Let $\mathfrak{g}_{+}, \mathfrak{g}_{-}$defined as in Appendix $C$ and define the cone $V$ to consist of those $X$ in $\mathfrak{g}_{\text {- }}$ which can be written in the form $X=-\tau u H u^{-1}$ for a $\tau>0, u \in \mathfrak{U}$. Then all $\Lambda$ of the form (4.4) may also be written in the form

$$
\mathfrak{\varsigma}^{0}: \Lambda=u^{\prime} e^{X}, \quad u^{\prime} \in \mathfrak{U}, \quad X \in V .
$$

Since we know that this is a semigroup, it coincides with that defined by (C.1).

The assertion of the theorem gives us our

Proposition 1. In any weakly conformal invariant Wightman quantum field theory with a unique vacuum, the Hilbert space of physical states carries a unitary representation of the universal covering group $\mathbf{6}^{*}$ of $\mathrm{SO}_{e}(4,2) / \mathbb{Z}_{2}$. The vacuum $\Omega$ is invariant and the conformal Hamiltonian" $H=J_{60}=\frac{1}{2}\left(P^{0}+K^{0}\right)$ is positive and self-adjoint, $H \geqq 0$. The vacuum $\Omega$ is the only eigenvector of $H$ to eigenvalue 0 .

The self-adjoint generators $J_{A B}$ of $\mathfrak{6}^{*}$ act on the state vectors $\Psi^{E}\left(\xi_{1} \ldots \xi_{n}\right)$ as differential operators which differ from the generators of the semigroup $\Xi$ only by factors of $i$, viz.

$$
J_{A B}=i \Sigma_{j}\left(\xi_{j A} \partial_{j B}-\xi_{j B} \partial_{j A}\right) ; \quad A, B=0 \ldots 3,56 ; \quad \partial_{j A}=\frac{\partial}{\partial \xi_{j}^{A}}, \quad \xi_{j 0} \equiv i \xi_{j}^{4} \quad \text { etc } .
$$

We recall: weak conformal invariance means that the Euclidean Green functions are invariant under $\mathrm{SO}_{e}(5,1)$. The assertion made about the vacuum are straightforward consequences of Definition (6.1), viz. invariance of $\Omega$ under $\widetilde{\Xi}$. and the conformal cluster property (5.1). The unitary representation operators will henceforth be denoted by $U(\Lambda), \Lambda \in \mathfrak{G}^{*}$.

\section{The Manifold $\tilde{M}$}

In Section 4 we have introduced hyperbolic coordinates on the cone $C_{5,1}^{+}(\mathbb{R})$, viz.

$$
\xi^{6}=r \operatorname{ch} \sigma, \quad \xi^{4}=r \operatorname{sh} \sigma, \quad \xi^{k}=r e^{k} \quad(k=123,5) .
$$

where $e=\left(e^{k}\right)$ is a unit 4 -vector. The Euclidean coordinates $\mathfrak{x}=\left(x^{4}, \boldsymbol{x}\right)$ become in this parametrization

$$
x^{4}=\frac{\operatorname{sh} \sigma}{\operatorname{ch} \sigma+e^{5}} ; \quad x=\frac{e}{\operatorname{ch} \sigma+e^{5}} .
$$


The Euclidean Green functions $G_{n}\left(\xi_{1} \ldots \xi_{n}\right)$ may be considered as functions of these coordinates

$$
G_{n}\left(\xi_{1} \ldots \xi_{n}\right)=G_{n}\left(r_{1} e_{1} \sigma_{1} \ldots r_{n} e_{n} \sigma_{n}\right)
$$

They depend on variables $r_{i}$ only through overall factors $r_{i}^{-d}$.

In the next section we will show that they can be analytically continued in variables $\sigma_{j}$ to pure imaginary values

$$
\sigma_{j}=i \tau_{j}, \quad-\infty<\tau_{j}<+\infty .
$$

Of course, the Euclidean Green functions depend on $\sigma$ only through $\operatorname{ch} \sigma$. This must not mislead the reader into believing that the result of the analytic continuation depends on $\tau$ only through $\cos \tau$, for in general the analytic continuation of a holomorphic function is defined on a Riemann surface with several sheets. We will find however that there is a schlichte (single-sheeted) domain of analyticity $\operatorname{Re} \sigma_{1}>\operatorname{Re} \sigma_{2}>\ldots>\operatorname{Re} \sigma_{n}$ and the formal limit as $\operatorname{Re} \sigma_{i} \rightarrow 0$ through this domain is a one-valued "function" of "time variables" $\tau_{1} \ldots \tau_{n}$ if it exists. The unit 4-vectors $e_{i}$ play the role of "space variables" in which no analytic continuation is to be done.

We are thus led to consider the manifold $\tilde{M}$ consisting of points $(e, \tau)$ viz.

$$
\tilde{M}=\{(e, \tau) ;-\infty<\tau<+\infty, e=\text { real unit } 4 \text {-vector }\} .
$$

In the following we will review some results of Todorov, Meyer and Go $[8,9]$ to the extent that they will be needed later on.

Minkowski space $M^{4}$ may be imbedded into $\tilde{M}$ by identifying it with a restricted set of points

$$
M^{4}=\left\{(e, \tau) \in \tilde{M} ;-\pi<\tau<\pi, e^{5}>-\cos \tau\right\} .
$$

The relation with the usual Minkowskian coordinates $x^{\alpha}$ is given by

$$
x^{0}=\frac{\sin \tau}{\cos \tau+e^{5}}, \quad \boldsymbol{x}=\frac{\boldsymbol{e}}{\cos \tau+e^{5}}
$$

by analytic continuation of (7.1). We see that there is a bijective correspondence between $M^{4}$ defined by (7.5) and Minkowski space $\left\{x^{\alpha}\right\}$. If we write $e^{5}=\cos \theta$, $\boldsymbol{e}=\sin \theta \boldsymbol{\varepsilon}$ then the line element becomes

$$
d x^{\alpha} d x_{\alpha}=(\cos \tau+\cos \theta)^{-2}\left\{d \tau^{2}-d \theta^{2}-\sin ^{2} \theta d \varepsilon d \varepsilon\right\}
$$

with $\varepsilon \cdot d \boldsymbol{\varepsilon}=0$ since $\boldsymbol{\varepsilon}^{2}=1$. We see that the surfaces $\tau=$ const are space-like within $M^{4}$ in the ordinary sense, viz.

$$
d x^{\alpha} d x_{\alpha}<0 \quad \text { if } \quad d \tau=0, \quad(e, \tau) \in M^{4} .
$$

It is known that the space $\tilde{M}$ can be considered as a homogenous space of the q.m. conformal group $\mathfrak{G}^{*}$, that is $\mathfrak{6}^{*}$ acts transitively as a group of transformations of $\tilde{M}$. To describe their action let us introduce projective coordinates

$$
\tilde{\eta}=(r, e, \tau) ; \quad r>0, \quad(e, \tau) \in \tilde{M} .
$$


We define an action of $\mathfrak{5}^{*}$ on $\tilde{\eta}$ which commutes with scaling $r \rightarrow \lambda r$. In particular the conformal Hamiltonian" $H=J_{60}$ generates translations of $\tau$ while $J_{i j}(i j=123,5)$ generate rotations of $e$. For other 1-parameter subgroups it is best to consider the space $\{\tilde{\eta}\}$ as an $\infty$-sheeted covering of the cone $C_{2,4}(\mathbb{R})$ which consists of real 6 -vectors $\eta=\left(\eta^{A}\right), A=0 \ldots 3,56$. To get it one cuts the cone along the line $\kappa \equiv \eta^{6}$ $+\eta^{5}=0$ and glues 2 consecutive sheets together along this line.

The space $\{\tilde{\eta}\}$ is mapped onto the cone by the projection $\pi: \tilde{\eta} \rightarrow \eta$, viz. $\pi=\left(\pi^{A}\right)$,

$$
\eta^{A}=\pi^{A}(\tilde{\eta}): \quad \eta^{6}=r \cos \tau, \quad \eta^{4}=r \sin \tau, \quad \eta^{k}=r e^{k} \quad(k=123,5) .
$$

The action of $\mathfrak{G}^{*}$ on the space $\tilde{M}$ is such that [8]

$$
\pi^{A}(\Lambda \tilde{\eta})=\Lambda_{B}^{A} \eta^{B}
$$

where $\Lambda^{A}{ }_{B}$ acts as a pseudorotation on the cone $C_{2,4}(\mathbb{R})$ in the usual way [viz. $\left(\Lambda^{A}{ }_{B}\right) \in \mathfrak{G}^{*} / \Gamma \simeq \mathrm{SO}_{e}(4,2)$ where $\Gamma \subset$ center of $\left.\mathfrak{5}^{*}\right]$.

Translations (generated by $P_{\mu}=J_{6 \mu}-J_{5 \mu}$ ), Lorentz-transformations (generated by $J_{\mu v} ; \mu, v=0 \ldots 3$ ) and dilatations (generated by $J_{56}$ ) leave invariant the individual sheets of the manifold $\{\tilde{\eta}\}$ over the cone. Keeping this in mind, their action is completely specified by the corresponding pseudorotation of the cone.

Arbitrary conformal transformations $\Lambda \in \mathbf{6 5}^{*}$ may be compounded from the special types of transformations considered so far.

It is also known from the work of Segal [7] that the mainfold $\tilde{M}$ admits a (5*-invariant global causal ordering $>$. It has been described e.g. by Todorov [9], viz.

$$
\left(e_{1}, \tau_{1}\right)>\left(e_{2}, \tau_{2}\right) \quad \text { iff } \tau_{2}-\tau_{1}>\operatorname{Arccos} e_{1} \cdot e_{2}
$$

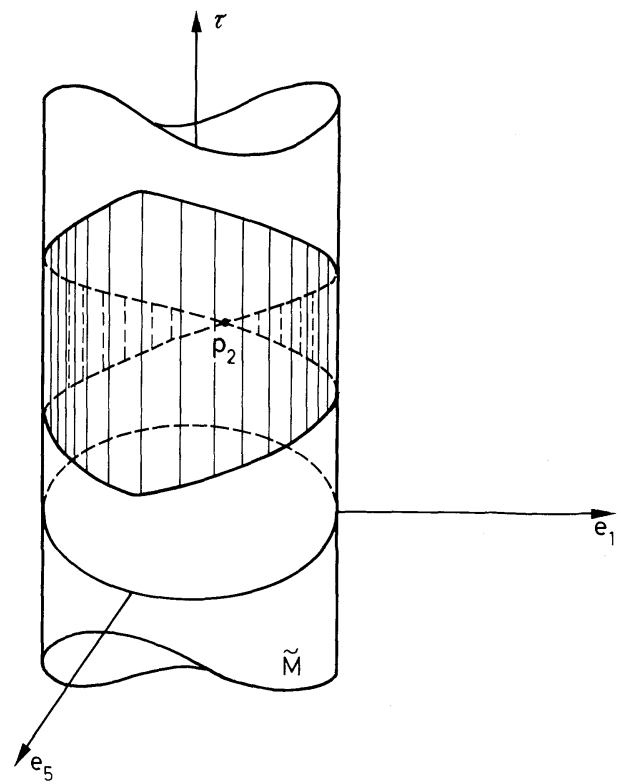

Fig. 2. Manifold $\tilde{M}$. Shaded part is $M^{4} \cdot p_{2}$ is the unique point at spatial infinity of $M^{4}$. Drawing for 2 space time dimensions 
where $\operatorname{Arccos} x$ is the principal value of $\arccos x$ which lies between $0 \ldots \pi .\left(e_{1}, \tau_{1}\right)$ and $\left(e_{2}, \tau_{2}\right)$ are relatively spacelike" if $\left|\tau_{2}-\tau_{1}\right|<\operatorname{Arccos} e_{1} \cdot e_{2}$ or, equivalently, if there exists a conformal frame where $\tau_{1}=\tau_{2}$. When restricted to Minkowskispace $M^{4}$, this causal ordering agrees with the usual one. It is important to note that the q.m. conformal group acts transitively on the set of pairs $\left(p_{1}, p_{2}\right)$ of relatively spacelike points $p_{1}=\left(e_{1}, \tau_{1}\right)$ and $p_{2}=\left(e_{2}, \tau_{2}\right)$. This is seen as follows: By a suitable rotation of $e$ and translation of $\tau$ we may take $p_{2}$ to $p_{2}=((\mathbf{0},-1), 0)$ i.e. the unique point at spatial infinity of $M^{4}$. Then the little group $\mathfrak{H}$ of $p_{2}$ consists of dilatations, Lorentz transformations and translations, and the set of points $p_{1}$ which are relatively spacelike to $p_{2}$ coincides with Minkowski space $M^{4}$ as defined in (7.5). But $\mathfrak{G}$ acts transitively on $M^{4}$ and therefore the asserted transitivity property follows.

\section{Analytic Continuation of Euclidean Green Functions}

Consider the state vectors $\Psi^{E}$ and Euclidean Green functions $G_{n}$ as functions of the hyperbolic coordinates, viz. $\Psi^{E}\left(\xi_{1} \ldots \xi_{m}\right)=\Psi^{E}\left(r_{1} e_{1} \sigma_{1} \ldots r_{m} e_{m} \sigma_{m}\right)$ and $G_{n}=G_{n}\left(r_{1} e_{1} \sigma_{1} \ldots r_{n} e_{n} \sigma_{n}\right)$ as defined in Eq. (7.2). Points $\xi$ where the hyperbolic coordinates are singular will be left out of consideration henceforth, i.e. we consider only finite values of $\sigma_{i}$ and $r_{i}>0$.

Let us smear with testfunctions $g\left(e_{1} \ldots e_{m}\right)$ resp. $h\left(e_{1} \ldots e_{n}\right)$. We define $(d \Omega$ $=$ usual measure on 4-dim. unit sphere $e \cdot e=1$ ),

$$
\Psi^{E}\left(\sigma_{1} \ldots \sigma_{m} \mid g\right)=\int d \Omega_{1} \ldots d \Omega_{m} g\left(e_{1} \ldots e_{m}\right) \Psi^{E}\left(r_{1} e_{1} \sigma_{1} \ldots r_{m} e_{m} \sigma_{m}\right)
$$

and

$$
\begin{aligned}
& G_{m+n}\left(\sigma_{1} \ldots \sigma_{m+n} \mid g h\right)=\left(\Psi^{E}\left(-\sigma_{m} \ldots-\sigma_{1} \mid g\right), \Psi^{E}\left(\sigma_{m+1} \ldots \sigma_{m+n} \mid h\right)\right) \\
& \quad=\int d \Omega_{1} \ldots d \Omega_{m+n} \bar{g}\left(e_{m} \ldots e_{1}\right) h\left(e_{m+1} \ldots e_{m+n}\right) G_{m+n}\left(r_{1} e_{1} \sigma_{1} \ldots r_{m+n} e_{m+n} \sigma_{m+n}\right) .
\end{aligned}
$$

Because of analyticity of Green functions the r.h.s. is well defined for $\sigma_{1}<\sigma_{2}<\ldots$ $<\sigma_{m}<0<\sigma_{m+1}<\ldots<\sigma_{m+n}$. Therefore also the vector $\Psi^{E}\left(\sigma_{1} \ldots \sigma_{m} \mid g\right)$ is a well defined vector in $\mathscr{H}$ with finite norm whenever $0<\sigma_{1}<\ldots<\sigma_{n}$ i.e. there comes no trouble from singularities of $\Psi^{E}$ at $x_{i}^{4}=x_{k}^{4}$ (Actually there are none, cp. end of Section 2).

Because of translational invariance in variables $\sigma_{i}$ the Green functions defined by Eq. (8.1b) depend in fact only on difference variables $\sigma_{k+1}-\sigma_{k}$ (The $r_{i}$-dependence is explicitly known and of no concern here). Moreover,

$$
\begin{aligned}
& \left(\Psi^{E}\left(-\sigma_{m} \ldots-\sigma_{1} \mid g\right), e^{-H s} \Psi^{E}\left(\sigma_{n+1} \ldots \sigma_{m+n} \mid h\right)\right) \\
& \quad=G_{m+n}\left(\sigma_{1} \ldots \sigma_{m}, \sigma_{m+1}+s \ldots \sigma_{m+n}+s \mid g h\right) \text { for } s \geqq 0
\end{aligned}
$$

since $H$ acts on state vectors $\Psi^{E}$ as a translation operator in $\sigma$. The analysis from here on is an exact replication of the analysis of Glaser [12] or of that part of [13] which does not use axiom $\left(E .0^{\prime}\right)$. It uses the classical Hille-Yosida theorem and the concept of envelope of holomorphy. The result is the following.

Proposition 2. In a weakly conformal invariant Wightman QFT (with a unique vacuum) the Euclidean Green functions $G_{n}\left(r_{1} e_{1} \sigma_{1} \ldots r_{n} e_{n} \sigma_{n}\right)$ can be analytically continued to complex variables $\sigma_{k}=s_{k}+i \tau_{k}$. They are holomorphic for $0<s_{1}<\ldots<s_{n}$, $\tau_{k}$ arbitrary real. 
We see that the space $\tilde{M}$ appears as a real boundary of the domain of analyticity of the Green functions, viz. $s_{k} \rightarrow 0$ for all $k$ through values such that $s_{k+1}>s_{k}>0$.

Example. Let us consider the 2-point function $G_{2}\left(\xi_{1} \xi_{2}\right)$ of a scalar field of dimension $d$. This is specified uniquely up to a normalization factor $n$ by weak conformal invariance. In the Euclidean domain

$$
G_{2}\left(\xi_{1} \xi_{2}\right)=n\left(\xi_{1} \xi_{2}\right)^{-d}=n\left(r_{1} r_{2}\right)^{-d}\left\{\operatorname{ch}\left(\sigma_{2}-\sigma_{1}\right)-e_{1} \cdot e_{2}\right\} .
$$

It is readily seen by inspection that this defines a holomorphic function of complex variables $\sigma_{1}=s_{1}+i \tau_{2}, \sigma_{2}=s_{2}+i \tau_{2}$ in the domain $0<s_{1}<s_{2}, \tau_{1}, \tau_{2}=-\infty \ldots+\infty$, because the expression in \{\} cannot vanish for such arguments. The limit as $s_{i} \rightarrow 0$ through values $s_{2}>s_{1}>0$ defines a tempered distribution $W_{2}\left(r_{1} e_{1} \tau_{1}, r_{2} e_{2} \tau_{2}\right)$. Formally

$$
W_{2}\left(r_{1} e_{1} \tau_{1}, r_{2} e_{2} \tau_{2}\right)=n\left(r_{1} r_{2}\right)^{-d}\left\{\cos \left(\tau_{2}-\tau_{1}-i 0\right)-e_{1} \cdot e_{2}\right\}^{-d} .
$$

Finally we may say something about the spectrum of $H$. Let $z=\exp \left(\sigma_{1}-\sigma_{2}\right)$. Then $|z|<1$ in the domain of holomorphy and expression (8.3) is proportional to the generating function for Gegenbauer-polynomials [9]. Therefore

$$
G_{2}\left(r_{1} \sigma_{1} e_{1}, r_{2} \sigma_{2} e_{2}\right)=n\left(\frac{1}{2} r_{1} r_{2}\right)^{-d} \sum_{k=0}^{\infty} e^{-\omega_{k}\left(\sigma_{2}-\sigma_{1}\right)} C_{k}^{d}\left(e_{1} \cdot e_{2}\right)
$$

with

$$
\omega_{k}=d+k
$$

Recalling that

$$
G_{2}(r e 0, r \sigma e)=\left(\Psi(r e 0), e^{-H \sigma} \Psi(r e 0)\right)
$$

we see that the Hilbertspace $\mathscr{H}$ contains eigenvectors of $H$ to eigenvalues $\omega_{k}=d+k$, $k=0,1,2, \ldots$ determined by the dimension $d$ of the scalar field.

This result can be generalized by considering the Euclidean Green functions obtained by analytically continuing the 2-point Wightman function (in Minkowski space) of a traceless symmetric tensor field $\Phi_{\alpha_{1} \ldots \alpha_{2}}(x)$. The Euclidean 2-point Green functions are again uniquely determined up to normalization by weak conformal invariance. They can be similarly expanded; the necessary computations will be done in Section 9. The result is as follows.

Suppose there exists in the original Wightman theory a local symmetric tensor field $\Phi_{\mu_{1} \ldots \mu_{s}}(x)$ with dimension $d_{s}$ and positive definite 2-point function - e.g. a current or stess tensor etc. Then the Hilbert space $\mathscr{H}$ contains eigenvalues of the conformal Hamiltonian $H$ to eigenvalues

$$
\omega_{k}=d_{s}+k, \quad k=0,1,2, \ldots .
$$

Lastly recall that the vacuum also is an eigenstate of $H$, with eigenvalue 0 (groundstate since $H \geqq 0$ ).

\section{Generalization to Arbitrary $\operatorname{Spin}^{7}$}

We now wish to discuss how the results obtained so far can be generalized to fields of arbitrary spin. There is just one point here which is not obvious. That is the choice of basis in index space. 
In a Wightman field theory in Minkowski space the fields are always assumed to obey the communication relations

$$
i \nabla^{\mu} \Phi_{\alpha}(x)=\left[\Phi_{\alpha}(x), P^{\mu}\right]
$$

with the generators of space time translations $P^{\mu}$. That is, these generators do not act on the indices. This amounts to fixing the choice of basis in index space at different points $x$ relative to each other. Of course for a theory with Poincare invariance as its only space time symmetry this is the evident and natural convention. However, for our purposes of analytically continuing from Minkowski space to the whole superworld $\tilde{M}$ it is an inappropriate choice and would lead to kinematical singularities. The reason for this will soon become clear. Instead, one can choose bases such that the new "conformal Hamiltonian" $H$ does not act on the indices. Formally

$$
i \frac{\partial}{\partial \tau} \Phi_{\alpha}(r e \tau)=\left[\Phi_{\alpha}(r e \tau), H\right]
$$

where $(r e \tau)=\tilde{\eta}$ are projective coordinates on $\tilde{M}$.

To be more explicit let us first recall [3] that all finite dimensional representations of the q.m. Lorentz group $\operatorname{SL}(2, \mathbb{C})$ can be constructed as tensor products of the two fundamental spinor representations. These representations can be analytically continued to representations of the complex Lorentz group and therefore in particular to (unitary) representations of the spin-covering of $\mathrm{SO}(4)$. All the Lorentz covariant fields may therefore be considered as multispinor fields to start with, viz.

$$
\Phi_{\alpha}^{n}(x) ; \quad \alpha=\left(\sigma_{1} \ldots \sigma_{j}, \dot{\tau}_{1} \ldots \dot{\tau}_{k}\right)
$$

if the field transforms according to the representation $\left(\frac{1}{2} j, \frac{1}{2} k\right)$. Different fields will be numbered by an index $n$. We label by $n^{*}$ the hermitean conjugate field, viz.

$$
\Phi_{\alpha^{*}}^{n^{*}}(x)=\Phi_{\alpha}^{n}(x)^{*}, \quad \alpha^{*}=\left(\tau_{1} \ldots \tau_{k}, \dot{\sigma}_{1} \ldots \dot{\sigma}_{j}\right) .
$$

Note that it transforms according to $\left(\frac{1}{2} k, \frac{1}{2} j\right)$. The Euclidean Green functions $G_{\alpha_{1} \ldots \alpha_{m}}^{n_{1} \ldots n_{m}}\left(\mathfrak{x}_{1} \ldots x_{m}\right)$ are obtained by analytically continuing the Wightman functions for such fields to the Euclidean domain.

The smeared Green functions

$$
G_{m}(f) \equiv \int d^{4} \mathfrak{x}_{1} \ldots d^{4} \mathfrak{x}_{m} G_{\alpha_{1} \ldots \alpha_{m}}^{n_{1} \ldots n_{m}}\left(\mathfrak{x}_{1} \ldots \mathfrak{x}_{m}\right) f_{\alpha_{1} \ldots \alpha_{m}}^{n_{1} \ldots n_{m}}\left(\mathfrak{x}_{1} \ldots \mathfrak{x}_{m}\right) .
$$

Summation over all repeated indices is understood.

The Euclidean time reversal operator $\theta$ acts on test functions according to

$$
\left(\theta f^{*}\right)_{\alpha_{1} \ldots \alpha_{m}}^{n_{1} \ldots n_{m}}\left(x_{1} \ldots \mathfrak{x}_{m}\right)=\bar{f}_{\alpha_{m}^{*} \ldots \alpha_{1}^{*}}^{n_{m}^{*} \ldots n_{1}^{*}}\left(\theta \mathfrak{x}_{m} \ldots \theta \mathfrak{x}_{1}\right) .
$$

With these conventions, the Euclidean Green functions obey positivity (2.14), viz.

$$
\sum_{k, l} G_{k+l}\left(\theta f_{k}^{*} \times f_{l}\right) \geqq 0
$$

for arbitrary finite sequences of test functions $f_{k} \equiv f_{\alpha_{1} \ldots \alpha k}^{n_{1} \ldots n k}\left(\mathfrak{x}_{1} \ldots \bar{x}_{k}\right) \in \mathscr{S}_{+}^{0}, k=0,1, \ldots$. So far everything is standard and valid for any Wightman QFT $[3,13]$. 
Let us now turn to the formulation of the hypothesis of weak conformal invariance. The transformation law (3.10) of test functions is replaced by [1]

$$
(\Lambda f)_{\alpha_{1} \ldots \alpha_{m}}^{n_{1} \ldots n_{m}}\left(\mathfrak{x}_{1} \ldots x_{m}\right)=D_{\alpha_{1} \beta_{1}}^{-\chi_{1}}\left(h_{1}\right) \ldots D_{\alpha_{m} \beta_{m}}^{-\chi_{m}}\left(h_{m}\right) f_{\beta_{1} \ldots \beta_{m}}^{n_{1} \ldots n_{m}}\left(\Lambda^{-1} \mathfrak{x}_{1} \ldots \Lambda^{-1} \mathfrak{x}_{m}\right)
$$

in $x$-space language. The notation is as follows: If $\chi=[l, \delta]$ then $-\chi=\left[l^{*}, 4-\delta\right]$, $l^{*}$ the complex conjugate representation to $l ; h_{i}$ are elements of the subgroup of stability $\mathfrak{H}$ of the point $\mathfrak{x}=0$ under the Euclidean conformal group $\mathfrak{5}=$ spin covering of $\mathrm{SO}_{e}(5,1)$.

The subgroup $\mathfrak{H}=\mathfrak{M} \mathfrak{M} \mathfrak{N}$ where $\mathfrak{M}$ consists of Euclidean Lorentz transformations [spin covering of $\mathrm{SO}(4)], \mathfrak{A}$ of dilatation, and $\mathfrak{N}$ of special conformal transformations. The little group elements $h_{i}$ are determined by $\mathfrak{x}_{i}$ and $\Lambda$ through

$$
h=t_{x}^{-1} \Lambda t_{x^{\prime}} \quad \text { with } \quad x^{\prime}=\Lambda^{-1} \mathfrak{x}
$$

and $t_{x}$ is the translation which takes 0 to $x$.

The matrices $D^{\chi_{2}}$ are specified by $\chi_{i}=\left[l_{i}, \delta_{i}\right]$ viz. spin" and dimension of the field $\Phi^{i}$. Explicitly, for $h=\operatorname{man}(m \in \mathfrak{M}$ etc.)

$$
D_{\alpha \beta}^{\chi}(\operatorname{man})=D_{\alpha \beta}^{l}(m) \sigma(a) \quad \text { with } \quad \sigma(a)=|a|^{-\delta}
$$

if $a$ is a dilatation by $|a| . D^{l}$ is the multi-spinor representation of the Euclidean Lorentz group discussed above.

For the scalar case, $D^{l}=1$ and the factors $\sigma(a)$ are eaten up upon going over to the $\xi$-space formulation.

The transformation law (9.8) is derived with the theory of induced representation; this has been explained in detail before $[1,6,20]$.

The hypothesis of weak conformal invariance asserts invariance of Euclidean Green functions as before, viz.

$$
G_{m}(\Lambda f)=G_{m}(f) \text { for all } \Lambda \text { in } \mathfrak{5} .
$$

Also the conformal cluster property (5.1) remains true in general, by the same arguments as in Section 5.

We have thus assembled all the ingredients which are necessary to guarantee the existence of a unitary representation of the q.m. conformal group $\mathfrak{5}^{*}$; proposition 1 holds thus in general.

We are not yet ready to carry out the analytic continuation of the Euclidean Green functions to $\tilde{M}$. The main problem is with the definition of the little group element $h$. Minkowskian space time translations $t_{x}$ leave invariant Minkowski space $M^{4}$ and can therefore not act transitively on the larger superworld $\tilde{M}$. They can therefore not be used to define little group elements for $\tilde{M}$.

We propose to remedy this by a (unitary) change of basis in index space. At the same time we go over to projective coordinates $\xi$ on Euclidean space.

Let $\hat{\xi}=\frac{1}{2}(\overrightarrow{0}, 11)$ a standard 6-vector, viz. $\hat{x}=0, \hat{\kappa}=1$. To every $\xi$ we may ${ }^{8}$ then select once and for all some standard boost $b_{\xi}$ of the form

$$
b_{\xi}=b_{\sigma} u a \text { such that } b_{\xi} \hat{\xi}=\xi \text {, }
$$

\footnotetext{
${ }^{8}$ We ignore subtleties associated with the points $\xi=(0, \pm r, 0, r), r>0$.
} 
with $u \in \mathfrak{U}$ and $a$ a dilatation. Explicitly, if

$$
\xi^{i}=\frac{1}{2} e^{i} \quad(i=123,5), \quad \xi^{4}=\frac{1}{2} \operatorname{sh} \sigma, \quad \xi^{6}=\frac{1}{2} \operatorname{ch} \sigma
$$

then $a$ is unity, $b_{\sigma}$ the $\sigma$-translation defined in Section 4 , and $u$ may be chosen in the form

$$
u=\left|\begin{array}{cc}
1-\frac{{ }^{t} \boldsymbol{e} \times \boldsymbol{e}}{1+e^{5}} & { }^{t} \boldsymbol{e} \\
-\boldsymbol{e} & e^{5}
\end{array}\right|
$$

$\boldsymbol{e}=\left(e^{1} e^{2} e^{3}\right),{ }^{t} \boldsymbol{e}$ its transpose (a column vector) and $\mathbb{1}$ the $3 \times 3$ unit matrix. Rows and columns are numbered 1235 from left to right and top to bottom.

Of course, if $x^{\alpha}=\xi^{\alpha} / \kappa$, then also

$$
t_{x} a^{\prime} \hat{\xi}=\xi
$$

for a suitable dilatation $a^{\prime}$, since $a^{\prime} \hat{\xi}=\left|a^{\prime}\right|^{-1} \hat{\xi}$.

Therefore $b_{\xi}^{-1} t_{x} a^{\prime}$ leaves invariance $\hat{\xi}$ and so belongs to its stability subgroup $\mathfrak{M N}$, viz.

$$
b_{\xi}^{-1} t_{x} a^{\prime}=m n \in \mathfrak{M} \mathfrak{M} .
$$

Of course $m, n$ depend on $\xi$. To find them one does not actually need to determine $a$, $a^{\prime}$, moreover $m$ depends on $\xi$ only through $\mathfrak{x}$. $\xi^{i}$ by

Let us now introduce new test functions which depend on projective coordinates

$f_{\alpha_{1} \ldots \alpha_{k}}^{\ldots}\left(\xi_{1} \ldots \xi_{k}\right)=\kappa_{1}^{d_{1}-4} \ldots \kappa_{k}^{d_{k}-4} f_{\beta_{1} \ldots \beta_{k}}\left(x_{1} \ldots \mathfrak{x}_{k}\right) D_{\beta_{1} \alpha_{1}}^{l_{1}}\left(m_{1}^{-1}\right) \ldots D_{\beta_{k} \alpha_{k}}^{l_{k}}\left(m_{k}^{-1}\right)$

with $m_{k}$ determined by $\xi_{k}$ through Eq. (9.16). Similarly

$G_{\alpha_{1} \ldots \alpha_{k}}^{\ldots}\left(\xi_{1} \ldots \xi_{k}\right)=\kappa_{1}^{-d_{1}} \ldots \kappa_{k}^{-d_{k}} D_{\alpha_{1} \beta_{1}}^{l_{1}}\left(m_{1}\right) \ldots D_{\alpha_{k} \beta_{k}}^{l_{k}}\left(m_{k}\right) G_{\beta_{1} \ldots \beta_{k}}^{\ldots}\left(x_{1} \ldots x_{k}\right)$.

They are homogeneous functions of the $\xi_{i}$. Instead of $D^{l}(m)$ one may also write $D^{\chi}\left(b_{\xi}^{-1} t_{x} a^{\prime}\right)$ since $D^{\chi}(m n)=D^{l}(m)$. With this notation, $G_{k}(f)$ as defined by Eq. (9.6) may also be written as

$$
G_{k}(f)=\int d \mu\left(\xi_{1}\right) \ldots d \mu\left(\xi_{k}\right) f_{\alpha_{1} \ldots \alpha_{k}}^{n_{1} \ldots n_{k}}\left(\xi_{1} \ldots \xi_{k}\right) G_{\alpha_{1} \ldots \alpha_{k}}^{n_{1} \ldots n_{k}}\left(\xi_{1} \ldots \xi_{k}\right) .
$$

The transformation law of the new testfunctions follows from (9.8) as

$(\Lambda f)_{\alpha_{1} \ldots \alpha_{k}}^{n_{1} \ldots n_{k}}\left(\xi_{1} \ldots \xi_{k}\right)=f_{\beta_{1} \ldots \beta_{k}}^{n_{1} \ldots n_{k}}\left(\Lambda^{-1} \xi_{1} \ldots \Lambda^{-1} \xi_{k}\right) D_{\beta_{1} \alpha_{1}}^{l_{1}}\left(h_{1}^{\prime-1}\right) \ldots D_{\beta_{k} \alpha_{k}}^{l_{k}}\left(h_{k}^{\prime-1}\right)$

with $h_{k}^{\prime}=b_{\xi_{k}}^{-1} \Lambda b_{\xi_{k}^{\prime}}, \xi_{k}^{\prime}=\Lambda^{-1} \xi_{k}, D^{l}(h) \equiv D^{l}(m)$ for $h=m n \in \mathfrak{M} \mathfrak{N}$.

This is the correct transformation law for an induced representation on the cone $C_{5,1}^{+} \simeq \mathfrak{6} / \mathfrak{M N}$ with $b_{\xi}$ as standard boost.

Moreover

$$
\left(\theta f^{*}\right)_{\alpha_{1} \ldots \alpha_{k}}^{n_{1} \ldots n_{k}}\left(\xi_{1} \ldots \xi_{k}\right)=\bar{f}_{\alpha_{k}^{*} \ldots \alpha_{1}^{*}}^{*} \ldots n^{*}\left(\theta \xi_{k} \ldots \theta \xi_{1}\right)
$$

since $D_{\alpha^{*} \beta^{*}}^{l^{*}}(m)=\bar{D}_{\alpha \beta}^{l}(\theta m \theta)$.

With the notation (9.18), positivity (2.14), weak conformal invariance (3.11) and conformal cluster property (5.1) remain valid as they stand. In addition, the new Green functions depend on the hyperbolic variables $\sigma_{i}$ [cp. Definition (4.1)] 
only through their differences $\sigma_{i+1}-\sigma_{i}$ as was true in the scalar case and was used in carrying out the analytic continuation of Euclidean Green functions in Section 8.

To verify this assertion, let us inspect the transformation law (9.19a) for the special case of a $\sigma$-translation: $\Lambda=b_{\sigma}$. It is readily verified that the little group elements

$$
h^{\prime}=b_{\xi}^{-1} b_{\sigma} b_{\xi^{\prime}}=1 \quad\left(\xi^{\prime}=b_{\sigma}^{-1} \xi\right) .
$$

Checking this is simplified by noting that we know a priori that $h^{\prime} \in \mathfrak{M} \mathfrak{N}$ therefore $h^{\prime} \in \mathfrak{U}$ implies $h^{\prime}=1$.

Thus, $\sigma$-translations do not act on the indices anymore. It follows then from weak conformal invariance that the Green functions may depend on variables $\sigma_{i}$ only through their differences.

With this, all the ingredients are ready which are necessary for the analytic continuation of the Euclidean Green functions in the variables $\sigma_{i}$ to $\tilde{M}$. Proposition 2 follows as before and holds therefore in general.

Example. Let $\Phi_{\alpha}(x)$ a traceless symmetric tensor field of $\operatorname{rank} l$ with dimension $\delta$, where $\alpha=\left(\alpha_{1} \ldots \alpha_{l}\right)$. Its Euclidean two point function in the old basis is obtained from $\left(\Omega, \Phi_{\alpha}^{*}\left(x_{1}\right) \Phi_{\beta}\left(x_{2}\right) \Omega\right)$ by analytic continuation to imaginary time and will be temporarily denoted by $\Delta_{\alpha \beta}^{\chi}\left(\mathfrak{x}_{1} \mathfrak{x}_{2}\right), \chi=[l, \delta]$.

It is determined by weak conformal invariance up to normalization; an explicit expression was given in [1].

There is an alternative and equivalent expression for $\Delta^{\chi}$ which is due to Koller [20]. The central role in it is played by a special element $\mathscr{R}$ of $\boldsymbol{6}$, known as a representative of the nontrivial element of the Weyl group.

$$
\mathscr{R}=\text { rotation by } \pi \text { in the } 45 \text {-plane . }
$$

It follows that $\mathscr{R} m \mathscr{R}^{-1}=\theta m \theta^{-1}$ for $m \in \mathfrak{M}$. We can extend the tensor representation $D^{l}$ of $\mathfrak{M}$ to a representation of the group $\mathfrak{M}^{\prime} \simeq \mathbf{0}(4)$ which is obtained by adjoining $\theta$ (or $\mathscr{R}$ ) to $\mathfrak{M}$. Kollers formula reads

$$
\Delta_{\alpha \beta}^{\chi}(x, 0)=n D_{\alpha \gamma}^{\chi}\left(t_{x}^{-1} \mathscr{R} t_{\mathscr{R} x}\right) D_{\gamma \beta}^{l}(\theta)
$$

$n$ is a normalization factor.

From this we obtain the propagator in the $\xi$-space language (new basis). Let $\hat{\xi}=\frac{1}{2}(\overrightarrow{0}, 11)$ as before and suppose for a moment that $\xi$ is such that $\xi^{\alpha}=x^{\alpha}$, $\kappa=1$. Then $a^{\prime}=1$ in (9.15) and therefore by Definition $(9.17 \mathrm{~b})$

$$
\begin{aligned}
\Delta_{\alpha \beta}^{\chi}(\xi, \hat{\xi}) & =n D_{\alpha \beta}^{\chi}\left(b_{\xi}^{-1} t_{x}\right) \Delta_{\alpha \beta}^{\chi}(x, 0) \\
& =n D_{\alpha \beta}^{\chi}\left(b_{\xi}^{-1} \mathscr{R} t_{\mathscr{R} x}\right) D_{\gamma \beta}^{l}(\theta) \\
& =n \sigma(a) D_{\alpha \beta}^{l}(m \theta) \text { for } b_{\xi}^{-1} \mathscr{R} t_{\mathscr{R} x}=\text { man } .
\end{aligned}
$$

In fact this formula is valid for general $\xi$ since both sides can be shown to have the same homogeneity properties in $\xi$. Of course $x^{\alpha}=\xi^{\alpha} / \kappa$ then.

It only remains to determine $m, a$ as functions of $\xi$. This is done by explicit computation with matrices. Koller has explained in great detail how such computations are done [20]. Let $\xi$ given by (9.13) viz.

$$
\xi^{i}=\frac{1}{2} e^{i} \quad(i=1235), \quad \xi^{4}=\frac{1}{2} \operatorname{sh} \tau, \quad \xi^{6}=\frac{1}{2} \operatorname{ch} \tau .
$$


Then $a$ is a dilatation by $|a|$

$|a|=\frac{1}{2}\left(\operatorname{ch} \sigma-e^{5}\right), \quad m^{\prime} \equiv m \theta=\frac{1}{2}|a|^{-1}\left|\begin{array}{ll}2|a| \mathbb{1}-{ }^{t} \boldsymbol{e} \times \boldsymbol{e} \frac{1+\operatorname{ch} \tau}{1+e^{5}} & -{ }^{t} \boldsymbol{e} \operatorname{sh} \tau \\ \boldsymbol{e} \operatorname{sh} \tau & 1-e^{5} \operatorname{ch} \tau\end{array}\right|$.

Hence finally

$$
\left.\Delta_{\alpha \beta}^{\chi}(\xi, \hat{\xi})=n^{\prime}\left(\operatorname{ch} \sigma-e^{5}\right)^{-\delta} S\left\{m_{\alpha_{1} \beta_{1}}^{\prime} \ldots m_{\alpha_{l} \beta_{l}}^{\prime}\right\}-\text { traces }\right)
$$

where $S$ acts as a symmetrizer in all arguments $\alpha_{i}, \beta_{i}$ separately, and $n^{\prime}=2^{\delta} n$ is a normalization factor.

\section{Epilogue}

Having established the analyticity of Green functions in variables $\sigma_{k}$ we can formally define the Wightman functions on the superworld $\tilde{M}$ by

$$
W_{n}\left(\tilde{\eta}_{1} \ldots \tilde{\eta}_{n}\right)=\lim _{\substack{s_{i} \rightarrow 0 \\ 0<s_{1}<\ldots<s_{n}}} G_{n}\left(r_{1} e_{1} s_{1}+i \tau_{1}, \ldots, r_{n} e_{n} s_{n}+i \tau_{n}\right) \text { for } \tilde{\eta}_{i}=\left(r_{i}, e_{i}, \tau_{i}\right) \text {. }
$$

They depend on $r_{i}$ only through an overall factor $r_{i}^{-d}$ by homogeneity of $G_{n}$, and they will be invariant under the q.m. conformal group $\mathfrak{G}^{*}$, viz.

$$
W_{n}\left(\Lambda \tilde{\eta}_{1} \ldots \Lambda \tilde{\eta}_{n}\right)=W_{n}\left(\tilde{\eta}_{1} \ldots \tilde{\eta}_{n}\right)
$$

The action of $\Lambda$ on points $\tilde{\eta}$ was described in Section 7 .

One would like to show that the Wightman functions formally defined by Eq. (10.1) are tempered distributions or at least generalized functions admitting of a test function space whose intersection with $\mathscr{D}\left(\tilde{M}^{n}\right)(\infty$ differentiable test functions with compact support) is dense therein. This is a difficult technical problem which we have not investigated.

However, in the study of models one has usually more information at hand. Let us therefore assume for further discussion that $W_{n}\left(\tilde{\eta}_{1} \ldots \tilde{\eta}_{n}\right)$ are indeed generalized functions in the sense just explained. The Gelfand-Naimark-Segal reconstruction theorem [3] then supplies us with fields $\phi(\tilde{\eta})$. They become operators with a common dense domain of definition after smearing with test functions; and $\phi(r, e, \tau)$ depends on $r$ only through a factor of $r^{-d}$. The fields are covariant in the sense that

$$
U(\Lambda) \phi(\tilde{\eta}) U(\Lambda)^{-1}=\phi(\Lambda \tilde{\eta})
$$

In particular

$$
i \frac{\partial}{\partial \tau} \phi(r, e, \tau)=[\phi(r, e, \tau), H]
$$

where $H \geqq 0$ is the conformal Hamiltonian constructed in Section 6 .

This equation of motion has two outstanding features. First, the dependence on $r$ of $\phi$ is trivial and the spatial variables $e$ form a compact space - they are unit 4-vectors. The usefulness of this observation is limited though by the fact that the fields are not operators yet after smearing only in the space variables. 
Second, the Hamiltonian $H$ has many discrete eigenvalues which are fixed by the dimensions of the local fields in the (Wightman) theory. This has been explained in Section 8. There is some indications (but no complete proof yet) that in theories which admit operator product expansions, $H$ has a purely discrete spectrum specified completely by the dimensions of the local fields in the theory.

Lastly, let us turn to the locality properties of the fields. We claim that

$$
\left[\phi\left(\tilde{\eta}_{1}\right), \phi\left(\tilde{\eta}_{2}\right)\right]_{-}=0 \quad \text { whenever } \tilde{\eta}_{1}, \tilde{\eta}_{2} \text { are relatively spacelike }
$$

in the sense explained in Section 7. Clearly this is true when $\tilde{\eta}$, and $\tilde{\eta}_{2}$ are projective coordinates of 2 relatively spacelike points in Minkowski space. This is one of the Wightman axioms which we assumed. But then also

$$
U(\Lambda)\left[\phi\left(\tilde{\eta}_{1}\right), \phi\left(\tilde{\eta}_{2}\right)\right] U(\Lambda)^{-1}=\left[\phi\left(\Lambda \tilde{\eta}_{1}\right), \phi\left(\Lambda \tilde{\eta}_{2}\right)\right]=0
$$

for arbitrary $\Lambda \in \mathfrak{G}^{*}$. Since $\mathfrak{G}^{*}$ acts transitively on pairs of relatively spacelike points $\left(e_{1}, \tau_{1}\right) \neq\left(e_{2}, \tau_{2}\right)$ of $\tilde{M}$, Eq. (10.4) follows.

Lastly we would like to refer to a recent result by Schroer and Swieca. It concerns the field transformation law under the center of $\mathfrak{6}^{*}$.

It follows from the work of [8] that the center of $\mathfrak{6}^{*}$ is a discrete abelian group isomorphic to $\mathbb{Z} \times \mathbb{Z}_{2}$. The factor $\mathbb{Z}_{2}$ leaves invariant the points of $\tilde{M}$ and serves to distinguish between bosons and fermions. The remaining factor

$$
\begin{gathered}
\mathbb{Z}=\left\{Z^{n} ; n=0, \pm 1, \pm 2, \ldots \text { with } Z=e^{i \pi H} \Pi\right\} \\
\Pi=4 \text {-rotation of } e \text { into }-e .
\end{gathered}
$$

Therefore $\Pi^{2}=1$ and

$$
\mathbb{Z}^{2 n}=e^{2 i \pi n H}
$$

Schroer and Swieca have pointed out [10] that in general

$$
U(Z) \phi(\tilde{\eta}) U(Z)^{-1} \neq z \phi(\tilde{\eta})
$$

for any phase factor $z$, and the same is still true if we consider positive and negative frequency part of $\phi$ separately. This means [cp. (10.3)] that fields with arguments over the same point $x$ of Minkowski-space do not just differ by a phase, and they transform under the center of $\mathfrak{6}^{*}$ in an essentially nontrivial way.

This conclusion was reached in [10] by study of a soluble 2-dimensional model. ${ }^{9}$ However it is valid more generally as we will now argue.

Starting point is the fact that we have some information about the dimensions of the composite fields in the theory: they are in general anomalous and dynamically determined.

Consider for instance $\phi^{3}$-theory in $D$ dimensions. [This theory is sick, but never mind....] Let $d \equiv \frac{1}{2} D-1+\Delta$ the dimension of the fundamental field. There ought to be symmetric tensor fields $O_{\alpha_{1} \ldots \alpha_{s}}(x)$ of arbitrary even rank $s=2,4, \ldots$ with dimension $d_{s} \equiv D-2+s+\sigma_{s}$. By positivity $[1,21,22] \sigma_{s}$ must be a (convex) function of $s$ satisfying $\sigma_{s+2} \geqq \sigma_{s} \geqq 0$ and $\sigma_{2}=0$ (stress tensor), $\sigma_{s} \rightarrow 2 \Delta$ as $s \rightarrow \infty$. The last relation was first discovered by one of the present authors [21]

9 Note added in manuscript: For the Thirring model the computation was done by Kupsch, Rühl and Yunn [33]. 
and was later proven by Callan and Gross in the more general frame work of summed up perturbation theory [23]. In conclusion, $\sigma_{s}$ have a nontrivial dependence on $s$.

Consider now the 3-point functions $\left(\Omega, \phi\left(x_{1}\right) \phi\left(x_{2}\right) O_{\alpha_{1} \ldots \alpha_{s}}\left(x_{3}\right) \Omega\right)$; they are not expected to vanish. The corresponding Euclidean Green functions are uniquely determined (up to normalization) by weak conformal invariance $[15,24]$ and can be analytically continued to $\tilde{M}$ as described in Section 9. Let $U \equiv U\left(Z^{2}\right)$. By (10.4b) and (9.2),

$$
\left(\Omega, U \phi\left(\tilde{\eta}_{1}\right) U^{-1} U \phi\left(\tilde{\eta}_{2}\right) U^{-1} O_{\alpha_{1} \ldots \alpha_{s}}\left(\tilde{\eta}_{3}\right) \Omega\right)=\left(\Omega, \phi\left(\tilde{\eta}_{1}\right) \phi\left(\tilde{\eta}_{2}\right) O_{\alpha_{1} \ldots \alpha_{s}}\left(Z^{-2} \tilde{\eta}_{3}\right) \Omega\right) .
$$

By inspection of the explicit expression for these 3-point functions one finds that this is

$$
=e^{-2 \pi i d_{s}}\left(\Omega, \phi\left(\tilde{\eta}_{1}\right) \phi\left(\tilde{\eta}_{2}\right) O_{\alpha_{1} \ldots \alpha_{s}}\left(\tilde{\eta}_{3}\right) \Omega\right)
$$

The phase factor herein depends on $s$ in a nontrivial way, and therefore equality in (10.5) cannot be true for any one $\%$.

Conversely it is clear from Eq. (10.4b) and the discussion in Section 8 of eigenvalues of $H$ that the unitary representation of the center of $\mathfrak{b}^{*}$ in the physical Hilbertspace contains a great deal of dynamical information already, in particular about the noninteger part of the dimensions of all local fields in the theory.

\section{Appendix A. Covariance Lemma}

Retain the notation of Section 3, in particular let the measure $d \mu(\xi)$ $=2 d^{6} \xi \delta(\xi \cdot \xi) \delta\left(\xi \cdot \eta^{-1}\right), \eta$ positive timelike or $\sim$ lightlike.

Lemma. Let $h(\xi)$ a locally integrable homogeneous function of $\xi, h(\varrho \xi)=\varrho^{-4} h(\xi)$ for $\varrho>0$ and such that $I=\int d \mu(\xi) h(\xi)$ exists (for some $\eta$ ). Then the integral $I$ is independent of the vector $\eta$ appearing in the definition of the measure $d \mu$, for $\eta$ positive lightlike or positive timelike.

Proof. Introduce hyperbolic coordinates $\xi=(r, e, \sigma)$ as in (4.1) and let $\hat{\xi}=r^{-1} \xi$ $=(1, e, \sigma)$. Working out the Jacobian we get

$$
d \mu(\xi)=2 r^{3} d r d \sigma d \Omega \delta(r \hat{\xi} \cdot \eta-1)
$$

with $d \Omega$ the usual measure on the 4-dimensional unit sphere $e \cdot e=1$. Integrating a homogeneous function $h(\xi)=r^{-4} h(\hat{\xi})$ we get

$$
\begin{aligned}
\int d \mu(\xi) h(\xi) & =\int d r d \sigma d \Omega 2 r^{-1} \cdot \delta(r \hat{\xi} \eta-1) h(\hat{\xi}) \\
& =2 \int d \sigma d \Omega h(\hat{\xi})
\end{aligned}
$$

which is independent of $\eta$. It is essential that $\eta$ is positive timelike or $\sim$ lightlike in order that $r$-integration can be trivially performed, for only then $\hat{\xi}_{\eta}>0$ except possibly for a set of measure zero.

The lemma has been used before in the literature on conformal QFT in $[9,15$, $18,21]$. For the group $\mathrm{SO}(3,1)$ it appears in the text book [25]. 


\section{Appendix B. Some Matrix Computations}

In this Appendix, the $6 \times 6$-matrix $\Lambda_{\tau}$ defined in (4.6) is calculated explicitly. Let us recall that to any euclidean conformal transformation there corresponds a $6 \times 6$-matrix $\Lambda \in \mathrm{SO}_{e}(5,1)$ (see Section 3 ). They are easily constructed for the following three cases

1) $b_{\tau}$ is defined by: $b_{\tau} \xi \equiv \xi^{\prime}$ where $\xi^{\prime 4}=\operatorname{ch} \tau \xi^{4}+\operatorname{sh} \tau \xi^{6} ; \xi^{\prime 6}=\operatorname{sh} \tau \xi^{4}+\operatorname{ch} \tau \xi^{6}$; $\xi^{\prime k}=\xi^{k} \quad(k=1,2,3,5)$.

Hence:

$$
b_{\tau}=\left|\begin{array}{|c|c|c|c|}
\mathbb{1} & 0 & 0 & 0 \\
\hline 0 & \operatorname{ch} \tau & 0 & \operatorname{sh} \tau \\
\hline 0 & 0 & 1 & 0 \\
\hline 0 & \operatorname{sh} \tau & 0 & \operatorname{ch} \tau
\end{array}\right| \mathbb{1} \equiv 3 \times 3 \text { unit matrix }
$$

2) The translations $t_{-\operatorname{sh} \tau}$ in the 4-direction (viz. $\left.t_{-\operatorname{sh} \tau} \mathfrak{x}=\left(x^{4}-\operatorname{sh} \tau, x\right)\right)$ correspond to:

$$
t_{-\operatorname{sh} \tau}=\left|\begin{array}{c|c|c|c|}
\mathbb{1} & 0 & 0 & 0 \\
\hline 0 & 1 & -\operatorname{sh} \tau & -\operatorname{sh} \tau \\
\hline 0 & \operatorname{sh} \tau & 1-\delta & -\delta \\
\hline 0 & -\operatorname{sh} \tau & \delta & 1+\delta
\end{array}\right|, \quad \delta \equiv \frac{1}{2}(\operatorname{sh} \tau)^{2}
$$

3) Dilatations $d_{\operatorname{ch} \tau}$ (viz. $\left.\left(d_{\operatorname{ch} \tau} \mathfrak{x}\right)^{\mu}=\operatorname{ch} \tau x^{\mu} ; \mu=1, \ldots, 4\right)$ take on the form:

$$
d_{\mathrm{ch} \tau}=\left|\begin{array}{|c|c|c|c|}
\mathbb{1} & & 0 & 0 \\
\hline 0 & 1 & 0 & 0 \\
\hline 0 & & \frac{1+\operatorname{ch}^{2} \tau}{2 \operatorname{ch} \tau} & \frac{1-\operatorname{ch}^{2} \tau}{2 \operatorname{ch} \tau} \\
\hline 0 & & \frac{1-\operatorname{ch}^{2} \tau}{2 \operatorname{ch} \tau} & \frac{1+\operatorname{ch}^{2} \tau}{2 \operatorname{ch} \tau}
\end{array}\right|
$$

$\Lambda_{\tau}$ is defined to be the product of the enumerated three matrices, namely:

$$
\Lambda_{\tau}=t_{-\operatorname{sh} \tau} d_{\mathrm{ch} \tau} b_{\tau}
$$

Performing the calculation yields:

$$
\Lambda_{\tau}=\mid \begin{array}{|c|c|c|c|}
\mathbb{1} & 0 & 0 & 0 \\
\hline 0 & \frac{1}{\operatorname{ch} \tau} & -\frac{\operatorname{sh} \tau}{\operatorname{ch} \tau} & 0 \\
\hline 0 & \frac{\operatorname{sh} \tau}{\operatorname{ch} \tau} & \frac{1}{\operatorname{ch} \tau} & 0 \\
\hline 0 & 0 & 0 & 1
\end{array} .
$$


This converges for $\tau \rightarrow \infty$ to

$$
\Lambda_{\infty}=\left|\begin{array}{|l|l|r|r|}
\mathbb{1} & 0 & 0 & 0 \\
\hline 0 & 0 & -1 & 0 \\
\hline 0 & 1 & 0 & 0 \\
\hline 0 & 0 & 0 & 1
\end{array}\right| .
$$

The half space $\left\{\mathfrak{x} \in \mathbb{R}^{4} \mid x^{4}>0\right\}=\left\{\xi \in C_{5,1}^{+} \mid \xi^{4}>0\right\}=\left\{\xi \in C_{5,1}^{+} \mid \eta \cdot \xi<0\right\}$ where $\eta=(\overrightarrow{0}, 1,0) \in \mathbb{R}^{6}$ is mapped by $\Lambda_{\tau}$ onto the set $\left\{\xi \in C_{5,1}^{+} \mid\left(\Lambda_{\tau} \eta\right) \cdot \xi<0\right\}=\left\{\xi \in C_{5,1}^{+} \mid\right.$ $\left.\xi^{4}+\operatorname{sh} \tau \xi^{5}>0\right\}=\left\{x \in \mathbb{R}^{4} \mid x^{2}-1-2 x^{4} / \operatorname{sh} \tau<0\right\}$ which is equal to the interior of the ball with center $(1 / \operatorname{sh} \tau, \mathbf{0})$ and radius $\operatorname{ch} \tau / \operatorname{sh} \tau$.

\section{Appendix C. Analytic Continuation of Contractive Lie Semigroup Representations}

Given a one parameter semigroup of self-adjoint contraction operators $T_{t}$ $(t \geqq 0)$ on a Hilbertspace $\mathscr{H}$, the Hille-Yosida theorem asserts the following [26]: Assume that $\left\|T_{t} \Psi-\Psi\right\| \rightarrow 0$ as $t \rightarrow 0$ for every $\Psi \in \mathscr{H}$, i.e. strong continuity at the identity. Then there exists a positive self-adjoint generator $H$ such that $T_{t}$ $=\exp (-H t)$. This implies among other things that the semigroup can be analytically continued to a 1 -parameter group of unitary operators exp $(-i H s)$.

We will generalize this theorem to a class of Lie-semigroups. The generalization is not entirely trivial because a representation of a Lie algebra by self-adjoint operators is not always integrable to a unitary representation of a corresponding Lie group.

We are interested in the following situation. Let $\mathbb{6}$ a Lie group with real Lie algebra $\mathfrak{g}$. Then every element $\Lambda$ in a neighborhood of the identity of $\mathfrak{G}$ can be written as $\Lambda=e^{X}$ for an $X$ in $\mathfrak{g}$. Let $\theta$ an automorphism of $\mathfrak{g}$ with $\theta^{2}=1$. This induces an automorphism of $\mathfrak{G}$ which ${ }^{10}$ will also be denoted by $\theta$. Let us split $\mathfrak{g}=\mathfrak{g}_{+}+\mathfrak{g}_{-}$, where $\mathfrak{g}_{+}$consists of all $X$ in $\mathfrak{g}$ such that $\theta(X)=X$, and $\theta(Y)=-Y$ for $Y$ in $\mathfrak{g}$. Then $\mathfrak{g}_{+}$is a subalgebra of $\mathfrak{g}$, and $\left[\mathfrak{g}_{+}, \mathfrak{g}_{-}\right] \subset \mathfrak{g}_{-},\left[\mathfrak{g}_{-}, \mathfrak{g}_{-}\right] \subset \mathfrak{g}_{+}$. It follows that $\mathfrak{g}^{*}=\mathfrak{g}_{+}+i \mathfrak{g}_{-}$is also a real Lie algebra. $\mathfrak{g}_{+}$is called a symmetric subalgebra of $\mathfrak{g}$ and $\mathfrak{g}^{*}$.

Let $\mathfrak{U}$ the connected subgroup of $\mathfrak{b}$ which is generated by $\mathfrak{g}_{+}$, and suppose that there exists an open convex cone $V \subset g_{-}$such that

i) $V$ is invariant under $\mathfrak{H}$, i.e. for every $u \in \mathfrak{H}, X \in V$ implies $u X u^{-1} \in V$.

ii) $V$ and $\mathfrak{g}_{+}$span the Lie algebra $\mathfrak{g}$.

We define

$$
\mathfrak{S}^{0}=\left\{\Lambda \in \mathfrak{G} ; \text { for a } \quad k \geqq 1 \exists X_{1} \ldots X_{k} \in V, u \in \mathfrak{U} \quad \text { such that } \quad \Lambda=e^{X_{1}} \ldots e^{X_{k}} u\right\} .
$$

Clearly $\mathfrak{S}^{0}$ is a connected semigroup contained in $\mathbf{6}$, since $V$ is invariant under $\mathfrak{U}$. It will follow from Lemma 1 below that $\mathfrak{S}^{0}$ has the same dimension as a manifold as $\mathfrak{6}$. The subgroup $\mathfrak{U} \subset \mathfrak{b}$ is not in $\mathfrak{\Im}^{0}$ but it consists of limit points of $\mathfrak{\Xi}^{0}$. We may therefore define another semigroup $\mathfrak{\Xi}^{\sim}=\mathfrak{\Xi}^{0} \cup \mathfrak{U}$. It shares with

\footnotetext{
${ }^{10}$ In the main text we wrote $\theta \Lambda \theta$ in place of $\theta(\Lambda)$.
} 
$\mathbb{S}^{0}$ the following stability property: If $\Lambda \in \mathbb{S}^{\sim}$ then also $\bar{\Lambda} \equiv \theta\left(\Lambda^{-1}\right) \in \mathfrak{S}^{\sim}$. We are now set to state our

Theorem. Let $\mathfrak{S}^{\sim} \mathcal{C} \mathfrak{5}$ a semigroup as described above, and $T$ a representation of $\mathfrak{\Im}^{\sim}$ by contraction operators on a Hilbert space $\mathscr{H}$, viz. $\|T(\Lambda)\| \leqq 1$ for all $\Lambda \in \mathbb{S}^{\sim}$. Suppose that

i) If $\Lambda \rightarrow 1$ through values in $\mathfrak{S}^{\sim}$ then also $\|T(\Lambda) \Psi-\Psi\| \rightarrow 0$ for all $\Psi$ in $\mathscr{H}$ (strong continuity at the identity).

ii) $(\Psi, T(\Lambda) \Phi)=(T(\bar{\Lambda}) \Psi, \Phi)$ with $\bar{\Lambda}=\theta\left(\Lambda^{-1}\right)$ for all $\Psi, \Phi$ in $\mathscr{H}$.

Then $T$ can be analytically continued to a unitary representation of the simply connected Lie group $\mathfrak{G}^{*}$ whose Lie algebra is $\mathrm{g}^{*}=\mathfrak{g}_{+}+i \mathfrak{g}_{-}$. It has the property that the selfadjoint generators $T(X)$ are positive for $-X \in V$.

Remarks. 1. The theorem might be of some help in the general representation theory of noncompact groups, for it yields analytic representations" of $\mathfrak{b}^{*}$ which are often difficult to obtain otherwise. In the present paper we use it to analytically continue a contractive representation of a maximal open semigroup $\mathfrak{S}^{0} \subset \mathfrak{G}$ $\simeq \mathrm{SO}(5,1)$ to a unitary representation of the universal covering group $\mathfrak{6}^{*}$ of $\mathrm{SO}(4,2)$. Contractive representations of $\mathbb{S}^{\sim}$ can be obtained in a heuristic manner from unitary representations of $\mathfrak{6}$ by splitting the representation space as in [17], and then analytically continuing in the continuous Casimir invariant. This idea will be further developed elsewhere [2].

2. By an analytic continuation of the representation $T(\cdot)$ of $\mathfrak{S}^{\sim}$ we mean the following: according to the classical Hille-Yosida-theorem, we may analytically continue the one parameter semigroup $T(\exp t X), t \geqq 0, X \in V$ to some unitary one parameter groups $T(\exp i \tau X), \tau \in \mathbb{R}$. Our theorem asserts that those unitary operators together with the unitary representation $T(u), u \in \mathfrak{U}$, of $\mathfrak{U}$ generate by finite multiplication a unitary representation of $\mathfrak{6 5}^{*}$. This also means that the infinitesimal generators of the new representation coincide with those given by $T(\Lambda), \Lambda \in \mathfrak{S}^{\sim}$, apart from some factors of $i$.

Before starting with the proof of the theorem, let us note that its hypotheses say in particular that the representation $T$ of $\mathbb{S}^{\sim}$ restricts to a unitary representation $T(u)$ of $\mathfrak{U} \subset \mathfrak{S}^{\sim}$. Moreover, it follows from continuity condition i) that also $\| T(\Lambda) \Psi$ $-T(u) \Psi \| \rightarrow 0$ whenever $\Lambda \rightarrow u \in \mathfrak{U}$ through values in $\mathfrak{S}^{\sim}$. [To see this define $\Lambda^{\prime}=u^{-1} \Lambda \in \mathbb{S}^{\sim}$ and note that $\|T(\Lambda) \Psi-T(u) \Psi\|=\left\|T\left(\Lambda^{\prime}\right) \Psi-\Psi\right\|$ by unitarity of $T(u)$.]

Proof of Theorem. We will first assemble three more or less well known lemmas:

Lemma 1. Let $X_{1}, \ldots, X_{n} \in \mathfrak{g}$ a basis of the Lie algebra $\mathfrak{g}$ and $\mathfrak{b}_{c}$ the local complexification of $\mathfrak{G}$, see [27]. Then there is an open, complex neighborhood $\mathcal{O} \subset \mathbb{C}^{n}$ such that the mapping

$$
\left(z_{1} \ldots z_{n}\right) \in \mathcal{O} \rightarrow \exp z_{1} X_{1} \cdots \exp z_{n} X_{n} \in \mathfrak{G}_{c}
$$

equips $\mathfrak{6}_{c}$ with an analytic parametrization of an open neighborhood of the identity 1 in $\mathfrak{G}_{c}$.

This lemma is an easy consequence of the fact that the indicated mapping is holomorphic in all variables [32] and has non-vanishing Jacobian at zero. By applying the implicit function theorem we get our result [14, Theorem 10.2.5]. 
Lemma $2[28,29]$. Let $\mathfrak{U}$ any Lie group and $T(\cdot)$ a continuous representation of $\mathfrak{U}$ by unitary operators in a Hilbert space. $\mathscr{H}$. Then there is a dense, linear subspace $\mathscr{D}_{\mathfrak{U}}$ of equianalytic vectors in $\mathscr{H}$, i.e. we have an open neighborhood.N of 1 in $\mathfrak{U}_{c}$, the local complexification of $\mathfrak{U}$, such that $T(x) \Phi, x \in \mathfrak{U}, \Phi \in \mathscr{D}$ may be analytically continued to all points of $\mathscr{N}$.

A point to be stressed here is the fact that $\mathscr{N}$ is independent of the vector $\Phi \in \mathscr{D}_{\mathfrak{u}}$, (see also [31] in this context).

Lemma 3 [30]. Let $\mathscr{A} \subset \mathbb{C}^{n}$ be an open set and $\Psi\left(z_{1} \ldots z_{n}\right) \in \mathscr{H}$ a vectorvalued function on it. Suppose furthermore, that $\left(\Phi, \Psi\left(z_{1} \ldots z_{n}\right)\right)$ is holomorphic in $\mathscr{A}$ for any $\Phi \in \mathscr{H}$. Then $\Psi\left(z_{1} \ldots z_{n}\right)$ is itself holomorphic in $\mathscr{A}$.

Now we are well enough equipped with lemmas to start with the proof of the theorem.

First, we choose a basis $X_{1}, \ldots, X_{n}$ of $g$ such that $X_{1}, \ldots, X_{l}$ belong to $V$ and $X_{l+1}, \ldots, X_{n} \in \mathfrak{g}_{+}$. Then Lemma 1 yields an open neighborhood $\mathcal{O} \subset \mathbb{C}^{n}$ of zero with the property that the mapping $\left(z_{1}, \ldots, z_{n}\right) \in \mathcal{O} \rightarrow \exp z_{1} X_{1} \ldots \exp z_{n} X_{n}$ $\equiv \exp ^{\prime}\left(z_{1}, \ldots, z_{n}\right)$ is an analytic parametrization of some open neighborhood of 1 in $\mathfrak{G}_{c}$, the local complexification of $\mathfrak{\mathfrak { 5 }}$.

Since $T(u), u \in \mathfrak{U}$, is a continuous, unitary representation of $\mathfrak{U}$, Lemma 2 gives us a dense set $\mathscr{D}_{\mathfrak{u}}$ of equianalytic vectors in $\mathscr{H}$. Of course we may find an open neighborhood $\mathcal{O}_{1}$ of zero in $\mathbb{C}^{n-l}$ such that $\mathcal{O}_{1} \subset \mathcal{O}$ and $\exp ^{\prime} \mathcal{O}_{1} \equiv\{x \in \mathfrak{U}$; $\left.x=\exp z_{l+1} X_{1} \cdots \exp z_{n} X_{n} ;\left(z_{l+1} \cdots z_{n}\right) \in \mathcal{O}_{1}\right\}$ is contained in $\mathcal{N}$, the open neighborhood of 1 in $\mathfrak{U}_{c}$ mentioned in Lemma 2. Given a vector $\Phi \in \mathscr{D}_{\mathfrak{u}}$, the vectors $T(u) \Phi$, $u \in \mathfrak{U}$ may be analytically continued to all points of $\mathcal{O}_{1}$.

The one parameter semigroups $T\left(\exp t X_{k}\right), t \geqq 0, k=1 \ldots l$ of selfadjoint contractions may be analytically continued to the half-plane $\mathbb{C}_{+}=\{z \in \mathbb{C} ; \operatorname{Re} z>0\}$. This follows from the classical Hille-Yosida Theorem [26]. Thus, for any $z \in \mathbb{C}_{+}$, there is a bounded operator $T\left(\exp z X_{k}\right)$ which coincides for real $z$ with $T\left(\exp t X_{k}\right)$ and has matrix elements $\left(\Psi, T\left(\exp z X_{k}\right) \Phi\right)$ which are holomorphic functions of $z$.

Let now $\Phi \in \mathscr{D}_{\mathfrak{u}}$ and $\Psi \in \mathscr{H}$ arbitrary. Then we may form the vectors $T\left(\exp z_{1} X_{1}\right)$ $\ldots T\left(\exp z_{l} X_{l}\right) T(u) \Phi \equiv \Phi_{z_{1} \ldots z_{n}}$ where $z_{i} \in \mathbb{C}_{+}, i=1 \ldots l$ and $u=\exp ^{\prime}\left(0 \ldots 0, z_{l+1} \ldots z_{n}\right)$ $\in \exp ^{\prime} \mathcal{O}_{1}$. For real $z_{1}, \ldots, z_{n}, \Phi_{z_{1} \ldots z_{n}}$ reduces to $T\left(\exp ^{\prime}\left(z_{1}, \ldots, z_{n}\right)\right) \Phi=T(\Lambda) \Phi$, $\Lambda \in \exp ^{\prime} \mathcal{O} \cap \mathbb{S}^{0}$. Moreover $F\left(z_{1} \cdots z_{n}\right)=\left(\Psi, \Phi\left(z_{1} \cdots z_{n}\right)\right)$ is analytic in each variable separately and therefore, using Hartog's theorem [33], is a holomorphic function in the open set $\mathscr{W}=\left(\mathbb{C}_{+}\right)^{l} \times \mathcal{O}_{1}$. Since $\Psi$ was arbitrary we conclude from Lemma 3 that $\Phi_{z_{1} \ldots z_{1}}$ is itself holomorphic in $\mathscr{W}$, and because $\mathscr{W} \cap \mathbb{R}^{n}$ is a real, nonempty neighborhood we are allowed to call $\Phi_{z_{1} \ldots z_{n}}$ the analytic continuation of $T(\Lambda) \Phi \Lambda$ $\epsilon\left(\exp ^{\prime} \mathscr{W}\right) \cap \mathfrak{S}^{0}$ to all of $\mathscr{W}$.

In the next step, we move $\mathscr{W}$ by redefinition of $\mathscr{D}_{\mathfrak{u}}$ in order to get as a domain of analyticity for $T(\Lambda) \Phi$ an open neighborhood $z \in \mathbb{C}^{n}$ of zero.

Let $X \in V$ sufficiently close to zero, so that $\exp X \in \exp ^{\prime} \mathscr{W}$. Then we define:

$$
\mathscr{D} \equiv\left\{\Psi \in \mathscr{H} ; \Psi=T(\exp X) \Phi \text { for some } \Phi \in \mathscr{D}_{\mathfrak{u}}\right\}
$$

Clearly $\mathscr{D}$ is a linear subspace of $\mathscr{H}$. It is also dense in $\mathscr{H}$, for if $(\chi, \Psi)=0$ for all $\Psi \in \mathscr{D}$, then $(\chi, T(\exp X) \Phi)=0, \forall \phi \in \mathscr{D}_{\mathfrak{U}}$ and hence $(T(\exp X) \chi, \Phi)=0$, $\forall \Phi \in \mathscr{D}_{\mathfrak{u}}$. Since $\mathscr{D}_{\mathfrak{u}}$ is dense in $\mathscr{H}$ we have $T(\exp X) \chi=0$ and consequently 


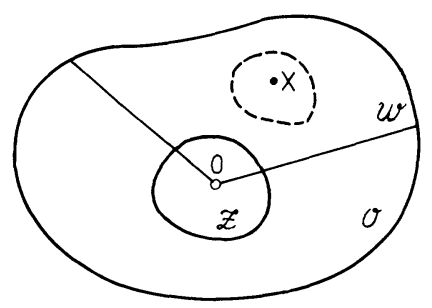

Fig. 3. Aera surrounded by dots: $\log ^{\prime}\left[\exp ^{\prime} \mathscr{Z} \cdot \exp X\right]$

$T(\exp t X) \chi=0, t \geqq 1$. By analyticity in $t$ and continuity for $t \downarrow 0$ it follows that $\chi=0$; hence $\mathscr{D}$ is dense.

Because of continuity of the group operations in $\mathfrak{6}_{c}$ there exists an open, connected neighborhood $\mathscr{Z} \subset \mathcal{O}$ of zero such that $\exp ^{\prime} \mathscr{Z} \cdot \exp X \subset \exp ^{\prime} \mathscr{W}$ (see Fig. 3).

$\mathscr{D}$ is defined in such a way that $T(\Lambda) \Psi, \Psi \in \mathscr{D}, \Lambda \in \exp ^{\prime} \mathscr{Z} \cap \mathbb{S}^{0}$ (this is open and nonempty in $\mathfrak{\Xi}^{0}$ ) may be analytically continued to all of $\mathscr{Z}$. Indeed, the vector $\Psi$ has the form $T(\exp X) \Phi, \Phi \in \mathscr{D}_{\mathfrak{u}}$, that is $T(\Lambda) \Psi=T(\Lambda \exp X) \Phi$. By $\left(z_{1}, \ldots, z_{n}\right)$ $\rightarrow \exp ^{\prime}\left(z_{1}, \ldots, z_{n}\right) \exp X$ where $\left(z_{1} \ldots z_{n}\right) \in \mathscr{Z}$ there is given an analytic parametrization of a complex neighborhood of $\exp X$ which is contained in $\exp ^{\prime} \mathscr{W}$. Hence $T(\Lambda \exp X) \Phi$ has an analytic continuation to all points of $\exp ^{\prime} \mathscr{Z} \exp X$, which just means that $T(\Lambda \exp X) \Phi$ defines a holomorphic function on $\mathscr{Z}$. This function is of course the analytic continuation of $T(\Lambda) \Psi$ to all of $\mathscr{Z}$. By uniqueness of analytic continuation $^{11}$, and because $T(\Lambda), \Lambda \in \mathfrak{S}^{0}$ is linear, we are able to define linear operators $T\left(\Lambda_{c}\right), \Lambda_{c} \in \exp \mathscr{Z} \subset \mathfrak{G}_{c}$, with domain of definition $\mathscr{D}$ by:

$$
T\left(\Lambda_{c}\right) \Psi \equiv \text { analytic continuation of } T(\Lambda) \Psi, \Lambda \in \exp ^{\prime} \mathscr{Z} \cap \mathbb{S}^{0}
$$

Now let $\mathscr{P} \subset \mathbb{C}^{n}$ be another complex neighborhood of zero with the following properties.

i) $\mathscr{P}$ is open and connected. The mapping $\left(z_{1}, \ldots, z_{n}\right) \in \mathscr{P} \rightarrow \exp \left(z_{1} X_{1}+\cdots\right.$ $\left.+z_{1} X_{n}\right) \equiv \exp \left(z_{1}, \ldots, z_{n}\right)$ is an analytic parametrization of a neighborhood of 1 in $\mathfrak{G}_{c}$.

ii) $\exp \mathscr{P} \cdot \exp \mathscr{P} \subset \exp ^{\prime} \mathscr{Z}$ which implies in particular that $T\left(\Lambda_{c}\right) \Psi, \Lambda_{c} \in \exp \mathscr{P}$, $\Psi \in \mathscr{D}$ is defined, and analytic in $\Lambda_{c}$.

iii) $\mathscr{P}=-\mathscr{P}, \mathscr{P}=\theta \mathscr{P}$ where $\theta\left(z_{1} \ldots z_{n}\right)=\left(-\bar{z}_{1} \ldots-\bar{z}_{l}, \bar{z}_{l+1} \ldots \bar{z}_{n}\right)(\bar{z}$ denotes the complex conjugate of $z$ ). Clearly $\theta^{2}=1$.

Such $\mathscr{P}$ always exists, and we may define a conjugation $\Lambda \in \exp \mathscr{P} \rightarrow \bar{\Lambda} \in \exp \mathscr{P}$ by: $\Lambda=\exp \left(z_{1}, \ldots, z_{n}\right) \Rightarrow \bar{\Lambda} \equiv \exp \left[-\theta\left(z_{1}, \ldots, z_{n}\right)\right]$. Whenever $\Delta \in \mathbb{S}^{0}, \bar{\Lambda}=\theta\left(\Lambda^{-1}\right)$ i.e. the definition of $\bar{A}$ coincides with the original one. Obviously the mapping $\Lambda \rightarrow \bar{\Lambda}$ is an antianalytic mapping of $\exp \mathscr{P}$ onto $\exp \mathscr{P}$.

Using once more uniqueness of analytic continuation we get the following two statements as consequences of the hypotheses of our theorem:

1) Let $\Lambda \in \exp \mathscr{P}$ and $\Phi, \Psi \in \mathscr{D}$. Then

$$
(\Phi, T(\Lambda) \Psi)=(T(\bar{\Lambda}) \Phi, \Psi)
$$

11 Recall that $\mathscr{Z}$ is connected and $\log ^{\prime}\left[\left(\exp ^{\prime} \mathscr{Z}\right) \cap \mathfrak{S}^{0}\right] \neq \phi$ is open in $\mathbb{R}^{n}$. 
2) Let $\Lambda_{1}, \Lambda_{2} \in \exp \mathscr{P}$ and $\Phi, \Psi \in \mathscr{D}$. Then

$$
\left(T\left(\overline{\Lambda_{1}}\right) \Phi, T\left(\Lambda_{2}\right) \Psi\right)=\left(\Phi, T\left(\Lambda_{1} \Lambda_{2}\right) \Psi\right) .
$$

Now we restrict ourselves to $\left(z_{1}, \ldots, z_{n}\right) \in \mathscr{P}$ with $\operatorname{Re} z_{i}=0$ for $i=1 \ldots l$ and $\operatorname{Im} z_{i}=0$ for $i=l+1 \ldots n$. These points correspond to an open neighborhood of 1 in $\mathfrak{5}^{*}$, viz. $\left(\mathfrak{b}^{*} \cap \exp \mathscr{P}\right.$. Furthermore $\bar{\Lambda}=\Lambda^{-1}$ for such $\Lambda$, and with 1$), 2$ ) and $T(1)=1$ we conclude, that $T(\Lambda)$ is a unitary operator which may be continuously extended to all of $\mathscr{H}$. We use the symbol $U(\Lambda)$ for these unitary operators. Because $U(\Lambda)$ is uniformly bounded and a strongly continuous function of $\Lambda \in \exp \mathscr{P} \cap \mathfrak{G}^{*}$ on $\mathscr{D}$, we conclude that $U(\Lambda)$ is strongly continuous on $\mathscr{H}$. Moreover we see from 2) that $U(\Lambda)$ builds up a unitary representation of the local group exp $\mathscr{P} \cap \mathfrak{G}^{*}$ which,following standard arguments [28], may be extended uniquely to a unitary representation of the whole, simply connected group $\mathfrak{5}^{*}$.

\section{Appendix D: A Technical Lemma}

Lemma. Let $\mathcal{O}$ an open set in $\mathbb{R}^{n}$ and $\mathscr{G}$ a real or complex domain ${ }^{12}$ containing $\mathcal{O}$. Let furthermore $\Phi\left(x_{1} \ldots x_{n}\right),\left(x_{1} \ldots x_{n}\right) \in \mathcal{O}$, a function on $\mathcal{O}$ with values in some Hilbert space $\mathscr{H}$ and assume that

$$
F\left(x_{1}^{\prime} \ldots x_{n}^{\prime}, x_{1} \ldots x_{n}\right) \equiv\left(\Phi\left(x_{1}^{\prime} \ldots x_{n}^{\prime}\right), \Phi\left(x_{1} \ldots x_{n}\right)\right)
$$

has an analytic continuation $F\left(z_{1}^{\prime} \ldots z_{n}^{\prime}, z_{1} \ldots z_{n}\right)$ to all of $\overline{\mathscr{G}} \times \mathscr{G}(\overline{\mathscr{G}}$ denotes the complex conjugate of $\mathscr{G})$. Then there is a vectorvalued, analytic function $\Phi\left(z_{1} \ldots z_{n}\right)$, $\left(z_{1} \ldots z_{n}\right) \in \mathscr{G}$, such that $\Phi\left(z_{1} \ldots z_{n}\right)=\Phi\left(x_{1} \ldots x_{n}\right)$ whenever $\left(z_{1} \ldots z_{n}\right)=\left(x_{1} \ldots x_{n}\right) \in \mathcal{O}$. Obviously, we have

$$
\left(\Phi\left(z_{1}^{\prime} \ldots z_{n}^{\prime}\right), \Phi\left(z_{1} \ldots z_{n}\right)\right)=F\left(\bar{z}_{1}^{\prime} \ldots \bar{z}_{n}^{\prime}, z_{1} \ldots z_{n}\right) .
$$

The lemma applies without further arguments to the function $\Psi^{E}\left(x_{1} \ldots x_{n}\right)$ (see Section 2): we just replace $\mathcal{O}$ by the set of all Euclidean points of the forward tube and $\mathscr{G}$ by the real domain $\left\{\left(x_{1} \ldots x_{n}\right) \in \mathbb{R}^{4 n} \mid x_{i}^{4} \geqq 0\right.$ (all $i$ ), $x_{i} \neq x_{j}$ (all $\left.\left.i \neq j\right)\right\}$. The function $F$ is then replaced by the Schwinger function $G_{2 n}\left(\theta x_{n}^{\prime} \ldots \theta x_{1}^{\prime}, x_{1} \ldots x_{n}\right)$, which is known to admit an analytic continuation to the extended, permuted tube, i.e. there is a domain of holomorphy for $G_{2 n}$ containing $\mathscr{G} \times \mathscr{G}$.

Proof of the Lemma. Because of infinite differentiability of $F\left(x_{1}^{\prime} \ldots x_{n}^{\prime}, x_{1} \ldots x_{n}\right)$ for arbitrary pairs of points $\left(x_{1}^{\prime} \ldots x_{n}^{\prime}\right),\left(x_{1} \ldots x_{n}\right) \in \mathcal{O}$ we conclude that $\Phi\left(x_{1} \ldots x_{n}\right)$ is an infinitely differentiable vector valued function, i.e. there are vectors

$$
D^{(v)} \Phi\left(x_{1}, \ldots, x_{n}\right) ; \quad D^{(v)} \equiv \frac{\partial^{v_{1}}}{\partial x_{1}^{v_{n}}} \ldots \frac{\partial^{v_{1}}}{\partial x_{n}^{v_{n}}} ; \quad\left(x_{1}, \ldots, x_{n}\right) \in \mathcal{O}
$$

with the property that

$$
\left(D^{\prime(\mu)} \Phi\left(x_{1}^{\prime} \ldots x_{n}^{\prime}\right), D^{(v)} \Phi\left(x_{1} \ldots x_{n}\right)\right)=D^{\prime(\mu)} D^{(v)} F\left(x_{1}^{\prime} \ldots x_{n}^{\prime}, x_{1} \ldots x_{n}\right) .
$$

The Cauchyformula for $F\left(z_{1}^{\prime} \ldots z_{n}\right)$ then yields an estimate for these derivatives:

$$
\left\|D^{(v)} \Phi\left(x_{1} \ldots x_{n}\right)\right\| \leqq M \nu ! \varrho^{\sum v_{\iota}} ; \quad v ! \equiv v_{1} ! \ldots v_{n} !
$$

12 I.e. an open, connected subset of $\mathbb{R}^{n}$ or $\mathbb{C}^{n}$. 
$\left[M, \varrho \in \mathbb{R}\right.$ are constants depending on $\left(x_{1} \ldots x_{n}\right)$ but not on $\left.v\right]$. Hence, the power series

$$
\sum_{(v)=0}^{\infty} \frac{1}{v !} D^{(v)} \Phi\left(x_{1} \ldots x_{n}\right)\left(z_{1}-x_{1}\right)^{v_{1}} \ldots\left(z_{n}-x_{n}\right)^{v_{n}}
$$

has positive radius of convergence and defines an analytic continuation of $\Phi\left(x_{1} \ldots x_{n}\right)$ to a complex neighborhood of $\mathcal{O}$.

We now make use of the principle of analytic continuation by overlapping polydiscs. Let $z \in \mathscr{G}$ and $x_{0} \in \mathcal{O}$. Then there are a sequence $a_{1}, \ldots, a_{m}$ of points of $\mathscr{G}, a_{1}=x, a_{m}=z$, and (open) polydiscs $P\left(a_{k}\right)$ such that

1) $\overline{P\left(a_{k}\right)} \times P\left(a_{k}\right) \subset$ domain of holomorphy of $F(k=1, \ldots, m)$,

2) $a_{k+1} \in P\left(a_{k}\right)(k=1, \ldots, m-1)$.

By repeating the above arguments it is easily seen that there exists an analytic continuation of $\Phi\left(x_{1}, \ldots, x_{n}\right),\left(x_{1} \ldots x_{n}\right) \in \mathcal{O} \cap P\left(a_{1}\right)$, to all of $\bigcup_{k=1}^{m} P\left(a_{k}\right)$ and that the value of this continuation at $z$ does not depend on the particular choice of the sequence $a_{1}, \ldots, a_{m}$.

\section{References}

1. Mack, G.: J. Phys. 34, Colloque C-1 (Suppl. au No 10) 99 (1973); In: Renormalization and Invariance in quantum field theory, E. R. Caianello (Ed.), New York: Plenum Press (1974)

2. Mack, G.: Lectures presented at the 1974 Bonn summer school. Proceedings: Lecture notes in physics. Berlin-Heidelberg-New York: Springer (in preparation)

3. Streater, R.F., Wightman, A.S.: PCT, Spin and Statistics, and all that. New York: W. A. Benjamin 1964

4. Hortacsu, M., Seiler, R., Schroer, B.: Phys. Rev. D5, 2518 (1972)

5. Kastrup,H.A., Mayer, D.H., Go,T.H., to be publ. in Rep. on Math. Phys. (1974)

6. Mack, G., Abdus Salam: Ann. Phys. (N.Y.) 53, 174 (1969)

7. Segal, I.: Bull. Am. Math. Soc. 77, 958 (1971)

8. Mayer, D. H.: Conformal invariant causal structures on pseudo-Riemannian manifolds. Preprint: Aachen, Apr. 1974

Go,T.H.: Some remarks on conformal invariant theories formulated on some four-Lorentz manifolds. Preprint: Aachen, June 1974

9. Todorov, I. T.: CERN TH 1697 (1973), Appendix

10. Schroer, B., Swieca, J.A.: Conformal transformations for quantized fields. Preprint: P. Univ. Catolica do Rio de Janeiro, Apr. 1974. See also: Swieca, J.A., Völkel, A.H.: Commun. math. Phys. 29, 319 (1973);

Rühl, W.: Preprint, Universität Trier-Kaiserslautern 1973;

Commun. math. Phys. 30, 287 (1973)

11. Misner, C. W., Thorne, K.S., Wheeler, J.A.: Gravitation. S. Francisco: Freeman 1973

12. Glaser, V.: CERN preprint TH 1706 (1973), to appear in Commun. Math. Phys.

13. Osterwalder, K., Schrader, R.: Commun. math. Phys. 31, 83 (1973) and in preparation;

Osterwalder, K.: In: Constructive Quantum Field Theory, G. Velo and A. S. Wightman (Eds.),

Lecture Notes in Physics 25, Verlag Berlin-Heidelberg-New York: Springer 1973

14. Dieudonné, J.: Foundations of modern analysis. New York: Academic Press 1969

15. Mack, G., Todorov, I. T.: Phys. Rev. D8, 1764 (1973)

16. Adler, S. L.: Phys. Rev. D6, 3445 (1972)

17. Ferrara, S., Mattioli, G., Rossi, G., Toller, M.: Nucl. Phys. B53, 366 (1973)

18. Kobayashi, S., Nomizu, K.: Foundations of differential geometry, Vol. 1, ch. I, propositions 4.2. New York: Interscience Publ. 1963

19. Gradshteyn, I. S., Ryzhik, I. M.: Table of integrals, series and products, formula 8.930. New York: Academic Press 1965

20. Koller, K.: DESY 74/8 and submitted to Commun. math. Phys. 
21. Mack, G.: In: Lecture Notes in Physics 17, p. 300, W. Rühl and A. Vancura (Eds.), Berlin- Heidelberg-New York: Springer 1973 (Sec. 8A)

22. Nachtmann, O.: Nucl. Phys. B63, 237 (1973)

23. Callan, C. G., Gross, D.: Phys. Rev. D8, 4383 (1973)

24. Ferrara,S., Gatto, R., Grillo, A.: Springer Tracts in Modern Physics, Vol. 67. Berlin-HeidelbergNew York: Springer 1973

25. Gelfand, I. M., Graev, M. I., Vilenkin, N. Ya., Generalized functions, Vol. 5, New York: Academic Press 1966

26. Hille, E., Phillips, R. S.: Functional Analysis and Semigroups, Amer. Math. Soc. Colloq. pub. 31, 1957

Reed,M.C.: In: Lecture Notes in Physics, Vol. 25, G. Velo and A. S. Wightman (Eds.). BerlinHeidelberg-New York: Springer 1973

27. Pontrjagin, L.: Topological groups. Princeton University Press 1946

28. Nelson, E.: Analytic vectors, Ann. Math. 70, 572 (1959)

29. Warner, G.: Harmonic analysis on semi-simple Lie groups, Vol. 1. Berlin-Heidelberg-New York: Springer 1972

30. Ref. 29, Lemma 4.4.5.1 and following remarks

31. Flato,M., Simon, J., Snellman,H., Sternheimer,D.: Simple facts about analytic vectors and analyticity, Ann. Sci. E.N.S. Paris 5 (3) 1972

Simon, J.: Commun. math. Phys. 23, 39 (1972)

32. Helgason, S.: Differential geometry and Symmetric Spaces, New York: Academic Press 1962

32. Bochner, S., Martin, W.T.: Several complex variables. Princeton Univ. Press 1948

33. Kupsch,J., Rühl, W., Yunn, B.C.: Conformal invariance of quantum fields in two-dimensional space-time, Universität Trier-Kaiserslautern, Preprint 1974

Communicated by R. Haag

\author{
M. Lüscher \\ Institut für \\ Theoretische Physik der Universität Bern \\ CH-3012 Bern, Switzerland \\ G. Mack \\ Institute for Advanced Study \\ Princeton, New Jersey 08540, USA
}

